Supplementary Material

\title{
Reaction of 3-(2-nitrophenyl)-1-arylprop-2-en-1-ones with triethylphosphite in microwave revisited: One-pot synthesis of 2-aroylindoles and 2-arylquinolines
}

\author{
Annah Gupta ${ }^{1}$, Rajni Khajuria ${ }^{1}$, Kamal K. Kapoor ${ }^{1}$ \\ ${ }^{1}$ Department of Chemistry, University of Jammu, Jammu 180006, India \\ E-mail: k2kapoor@yahoo.com
}

\section{EXPERIMENTAL}

\section{General}

All the commercially available reagents were purchased from Aldrich and were used without further purification. The reactions were performed with a Discover ${ }^{\mathrm{TM}}$ single mode cavity MW synthesizer (CEM Corporation). Melting points $\left({ }^{\circ} \mathrm{C}\right)$ were measured in open glass capillaries using Perfit melting point apparatus and are uncorrected. The progress of the reaction was monitored by thin layer chromatography (TLC) using silica gel pre-coated aluminium sheets (60 F254, Merck). Visualization of spots was effected by exposure to ultraviolet light (UV) at $254 \mathrm{~nm}$, iodine vapours, $2 \%$ 2,4-dinitrophenylhydrazine in methanol containing few drops of $\mathrm{H}_{2} \mathrm{SO}_{4}$ and draggendroff reagent. Column chromatography was performed on silica gel (60120 mesh). IR spectra $\left(v, \mathrm{~cm}^{-1}\right)$ were recorded on Perkin-Elmer FTIR spectrophotometer using $\mathrm{KBr}$ discs. ${ }^{1} \mathrm{H}$ NMR and ${ }^{13} \mathrm{C}$ NMR spectra in $\mathrm{CDCl}_{3}$ as solvents were recorded on Burker AC-400 spectrometer operating at $400 \mathrm{MHz}$ for ${ }^{1} \mathrm{H}$ and $100 \mathrm{MHz}$ for ${ }^{13} \mathrm{C}$, with tetramethylsilane (TMS) as internal standard. Electron impact mass spectra (EIMS) were recorded on Micro Mass VG-7070 $\mathrm{H}$ mass spectrometer at $70 \mathrm{ev.} \mathrm{Elemental} \mathrm{analysis} \mathrm{were}$ performed on Leco CHNS-932 analyzer. 


\section{General procedure}

A solution of 3-(2-nitrophenyl)-1-arylprop-2-en-1-ones $(1 \mathrm{mmol})$ in $\mathrm{P}(\mathrm{OEt})_{3}(6 \mathrm{mmol})$ in a capped vial was exposed to MW irradiation at $110{ }^{\circ} \mathrm{C}(80 \mathrm{~W})$. After the completion of the reaction (TLC), the reaction mixture was cooled to room temperature, transferred to a $10 \mathrm{~mL}$ round-bottomed flask and concentrated under vacuum at $80{ }^{\circ} \mathrm{C}$ in a rotary evaporator. The residue so obtained upon column chromatography yield pure products $\mathbf{2 ( a - i ; ~} \mathbf{5 7 - 6 2 \%}$ yield) and 3(a-i; 27-30\% yield).

\section{Spectral data of the synthesized compounds:}

(1H-indol-2-yl)(phenyl)methanone (2a) ${ }^{[1]}$ : Yellow solid, yield: $62 \%$, m.pt. $145-147{ }^{\circ} \mathrm{C}$ ( lit. 150-151 $\left.{ }^{\circ} \mathrm{C}\right),{ }^{1} \mathrm{H}$ NMR (400 MHz, $\left.\mathrm{CDCl}_{3}\right): \delta 9.65$ (br s, $\left.1 \mathrm{H}, \mathrm{NH}\right), 8.04(\mathrm{~d}, J=7.8 \mathrm{~Hz}, 2 \mathrm{H})$, $7.76(\mathrm{~d}, J=8.1 \mathrm{~Hz}, 1 \mathrm{H}), 7.66(\mathrm{t}, J=7.0 \mathrm{~Hz}, 1 \mathrm{H}), 7.60-7.52(\mathrm{~m}, 3 \mathrm{H}), 7.41(\mathrm{t}, J=7.6 \mathrm{~Hz}$, 1H), $7.20(\mathrm{t}, J=7.4 \mathrm{~Hz}, 2 \mathrm{H}) .{ }^{13} \mathrm{C} \mathrm{NMR}\left(100 \mathrm{MHz}, \mathrm{CDCl}_{3}\right): \delta 187.3,138.0,137.6,134.3$, $132.4,129.2,128.5,127.7,126.5,123.2,121.0,112.9,112.2 . \mathrm{IR}\left(\mathrm{KBr}, v_{\max } \mathrm{cm}^{-1}\right): 3315$, $1618,1577,1512,1328,1261,1132,734 . \mathrm{MS}(\mathrm{ESI})(\mathrm{m} / \mathrm{z}): 222$. Anal. calcd. for $\mathrm{C}_{15} \mathrm{H}_{11} \mathrm{NO}$ : C, 81.43; H, 5.01; N, 6.33\%. Found: C, 81.52; H, 5.02; N, 6.31\%.

(1H-indol-2-yl)(p-tolyl)methanone (2b) ${ }^{[1]}$ : Light brown solid, yield: $61 \%$, m.pt. 182-184 ${ }^{\circ} \mathrm{C}$ ( lit. $\left.189-190{ }^{\circ} \mathrm{C}\right),{ }^{1} \mathrm{H}$ NMR $\left(400 \mathrm{MHz}, \mathrm{CDCl}_{3}\right.$ ): $\delta 9.78$ (br s, $\left.1 \mathrm{H}, \mathrm{NH}\right), 7.97(\mathrm{~d}, J=7.9 \mathrm{~Hz}$, 2H), $7.75(\mathrm{~d}, J=8.1 \mathrm{~Hz}, 1 \mathrm{H}), 7.54(\mathrm{~d}, J=8.4 \mathrm{~Hz}, 1 \mathrm{H}), 7.40(\mathrm{dd}, J=14.2,7.4 \mathrm{~Hz}, 3 \mathrm{H}), 7.19$ $(\mathrm{d}, J=10.8 \mathrm{~Hz}, 2 \mathrm{H}), 2.50(\mathrm{~s}, 3 \mathrm{H}) .{ }^{13} \mathrm{C} \mathrm{NMR}\left(100 \mathrm{MHz}, \mathrm{CDCl}_{3}\right): \delta 187.0,143.1,137.5$, $135.3,134.5,129.4,129.2,127.7,126.3,123.1,120.9,112.5,112.3,21.7 . \mathrm{IR}\left(\mathrm{KBr}, v_{\max } \mathrm{cm}^{-}\right.$ $\left.{ }^{1}\right): 3307,1616,1510,1261,1170,1014,825,754 . \mathrm{MS}(\mathrm{ESI})(\mathrm{m} / \mathrm{z}): 236$. Anal. calcd. for $\mathrm{C}_{16} \mathrm{H}_{13} \mathrm{NO}: \mathrm{C}, 81.68 ; \mathrm{H}, 5.57 ; \mathrm{N}, 5.95$. Found: C, 81.54; H, 5.56; N, 5.97.

(1H-indol-2-yl)(4-methoxyphenyl)methanone (2c) ${ }^{[1]}$ : White solid, yield: 62\%, m.pt. 189$190{ }^{\circ} \mathrm{C}$ ( lit. $\left.190-191{ }^{\circ} \mathrm{C}\right),{ }^{1} \mathrm{H}$ NMR $\left(400 \mathrm{MHz}, \mathrm{CDCl}_{3}\right): \delta 9.58$ (br s, $\left.1 \mathrm{H}, \mathrm{NH}\right), 8.07$ (d, $J=8.5$ 
$\mathrm{Hz}, 2 \mathrm{H}), 7.75(\mathrm{~d}, J=8.1 \mathrm{~Hz}, 1 \mathrm{H}), 7.52(\mathrm{~d}, J=8.3 \mathrm{~Hz}, 1 \mathrm{H}), 7.39$ (t, $J=7.6 \mathrm{~Hz}, 1 \mathrm{H}), 7.23-$ $7.16(\mathrm{~m}, 2 \mathrm{H}), 7.06(\mathrm{~d}, J=8.5 \mathrm{~Hz}, 2 \mathrm{H}), 3.94(\mathrm{~s}, 3 \mathrm{H}) .{ }^{13} \mathrm{C} \mathrm{NMR}\left(100 \mathrm{MHz}, \mathrm{CDCl}_{3}\right): \delta 185.9$, $163.2,137.3,134.5,131.5,130.6,127.7,126.2,123.0,120.9,113.8,112.1,111.9,55.5$. IR $\left(\mathrm{KBr}, v_{\max } \mathrm{cm}^{-1}\right): 3296,1610,1510,1309,1257,1168,1026,833,758 . \mathrm{MS}(\mathrm{ESI})(\mathrm{m} / \mathrm{z}): 252$. Anal. calcd. for $\mathrm{C}_{16} \mathrm{H}_{13} \mathrm{NO}_{2}$ : C, 76.48; H, 5.21; N, 5.57\%. Found: C, 76.32; H, 5.20; N, $5.55 \%$.

(1H-indol-2-yl)(3-methoxyphenyl)methanone (2d) ${ }^{[1]}$ : Brown solid, yield: 58\%, m.pt. 125 $126^{\circ} \mathrm{C}$ ( lit. $\left.128-129^{\circ} \mathrm{C}\right),{ }^{1} \mathrm{H}$ NMR $\left(400 \mathrm{MHz}, \mathrm{CDCl}_{3}\right): \delta 9.93$ (br s, $\left.1 \mathrm{H}, \mathrm{NH}\right), 7.76(\mathrm{~d}, J=8.0$ Hz, 1H), $7.66(\mathrm{~d}, J=7.6 \mathrm{~Hz}, 1 \mathrm{H}), 7.58-7.54(\mathrm{~m}, 2 \mathrm{H}), 7.48(\mathrm{t}, J=7.9 \mathrm{~Hz}, 1 \mathrm{H}), 7.41(\mathrm{t}, J=$ $7.5 \mathrm{~Hz}, 1 \mathrm{H}), 7.25-7.18(\mathrm{~m}, 3 \mathrm{H}), 3.93(\mathrm{~s}, 3 \mathrm{H}) .{ }^{13} \mathrm{C} \mathrm{NMR}\left(100 \mathrm{MHz}, \mathrm{CDCl}_{3}\right): \delta 187.1,159.6$, $139.3,137.8,134.3,129.5,127.7,126.5,123.2,121.9,121.0,118.6,113.9,113.0,112.3$, 55.5. IR (KBr, $\left.v_{\max } \mathrm{cm}^{-1}\right): 3298,1614,1508,1312,1265,1159,1020,839,752 . \mathrm{MS}(\mathrm{ESI})$ $(\mathrm{m} / \mathrm{z}):$ 252. Anal. calcd. for $\mathrm{C}_{16} \mathrm{H}_{13} \mathrm{NO}_{2}: \mathrm{C}, 76.48 ; \mathrm{H}, 5.21 ; \mathrm{N}, 5.57 \%$. Found: $\mathrm{C}, 76.32 ; \mathrm{H}$, $5.22 ; \mathrm{N}, 5.56 \%$.

(4-Chlorophenyl)(1H-indol-2-yl)methanone (2e) ${ }^{[1]}$ : Light brown solid, yield: 58\%, m.pt. 194-196 ${ }^{\circ} \mathrm{C}$ ( lit. $\left.196-198{ }^{\circ} \mathrm{C}\right),{ }^{1} \mathrm{H}$ NMR $\left(400 \mathrm{MHz}, \mathrm{CDCl}_{3}\right): \delta 9.40$ (br s, 1H, NH), 7.97 (d, $J$ $=8.5 \mathrm{~Hz}, 2 \mathrm{H}), 7.75(\mathrm{~d}, J=8.1 \mathrm{~Hz}, 1 \mathrm{H}), 7.53(\mathrm{dd}, J=12.9,8.5 \mathrm{~Hz}, 3 \mathrm{H}), 7.44-7.40(\mathrm{~m}, 1 \mathrm{H})$, $7.23-7.17(\mathrm{~m}, 2 \mathrm{H}) .{ }^{13} \mathrm{C}$ NMR $\left(100 \mathrm{MHz}, \mathrm{CDCl}_{3}\right): \delta 185.8,138.8,137.6,136.2,134.0,130.6$, 128.8, 127.6, 126.8, 123.3, 121.2, 112.8, 112.2. IR (KBr, $\left.v_{\max } \mathrm{cm}^{-1}\right): 3307,1624,1504,1342$, 1259, 1089, 1014, 831, 754. MS(ESI) (m/z) : 256, 258. Anal. calcd. for $\mathrm{C}_{15} \mathrm{H}_{10} \mathrm{NOCl}: \mathrm{C}$, 70.46; H, 3.94; N, 5.48\%. Found: C, 70.59; H, 3.93; N, 5.50\%.

(4-Bromophenyl)(1H-indol-2-yl)methanone (2f): Light yellow solid, yield: 60\%, m.pt. 184-186 ${ }^{\circ} \mathrm{C},{ }^{1} \mathrm{H}$ NMR (400 MHz, $\mathrm{CDCl}_{3}$ ): $\delta 9.37$ (br s, $\left.1 \mathrm{H}, \mathrm{NH}\right), 7.90(\mathrm{~d}, J=8.4 \mathrm{~Hz}, 2 \mathrm{H})$, $7.73(\mathrm{dd}, J=16.8,8.3 \mathrm{~Hz}, 3 \mathrm{H}), 7.51(\mathrm{~d}, J=8.3 \mathrm{~Hz}, 1 \mathrm{H}), 7.42(\mathrm{t}, J=7.6 \mathrm{~Hz}, 1 \mathrm{H}), 7.20(\mathrm{dd}, J$ 
$=16.0,8.4 \mathrm{~Hz}, 2 \mathrm{H}) \cdot{ }^{13} \mathrm{C} \mathrm{NMR}\left(100 \mathrm{MHz}, \mathrm{CDCl}_{3}\right): \delta 185.9,137.6,136.7,133.9,131.8$, $130.72,127.7,127.3,126.8,123.3,121.2,112.8,112.1 . \mathrm{IR}\left(\mathrm{KBr}, \mathrm{v}_{\max } \mathrm{cm}^{-1}\right): 3309,1622$, 1512, 1327, 1263, 1134, 1010, 829, 752. MS(ESI) (m/z) : 300, 302. Anal. calcd. for $\mathrm{C}_{15} \mathrm{H}_{10} \mathrm{NOBr}: \mathrm{C}, 60.02 ; \mathrm{H}, 3.36$; N, 4.67\%. Found: C, 60.13; H, 3.35; N, 4.66\%.

(4-Fluorophenyl)(1H-indol-2-yl)methanone (2g) ${ }^{[1]}$ : White solid, yield: 60\%, m.pt. 183$185{ }^{\circ} \mathrm{C}$ ( lit. $\left.185-186{ }^{\circ} \mathrm{C}\right),{ }^{1} \mathrm{H}$ NMR $\left(400 \mathrm{MHz}, \mathrm{CDCl}_{3}\right): \delta 9.81$ (br s, $\left.1 \mathrm{H}, \mathrm{NH}\right), 8.08$ (dd, $J=$ 8.6, $5.5 \mathrm{~Hz}, 2 \mathrm{H}), 7.76(\mathrm{~d}, J=8.1 \mathrm{~Hz}, 1 \mathrm{H}), 7.54(\mathrm{~d}, J=8.3 \mathrm{~Hz}, 1 \mathrm{H}), 7.42(\mathrm{t}, J=7.6 \mathrm{~Hz}, 1 \mathrm{H})$, $7.28(\mathrm{~d}, J=2.9 \mathrm{~Hz}, 1 \mathrm{H}), 7.22(\mathrm{dd}, J=13.9,5.9 \mathrm{~Hz}, 3 \mathrm{H}) \cdot{ }^{13} \mathrm{C}$ NMR $\left(100 \mathrm{MHz}, \mathrm{CDCl}_{3}\right): \delta$ $185.8,166.6,164.1,137.7,134.1,131.7,127.6,126.6,123.2,121.1,115.8,115.6,112.8$, 112.3. IR (KBr, $\left.v_{\max } \mathrm{cm}^{-1}\right): 3311,1627,1508,1327,1247,1143,1010,833,756 . \mathrm{MS}(\mathrm{ESI})$ $(\mathrm{m} / \mathrm{z})$ : 240. Anal. calcd. for $\mathrm{C}_{15} \mathrm{H}_{10} \mathrm{NOF}: \mathrm{C}, 75.30 ; \mathrm{H}, 4.21 ; \mathrm{N}, 5.85 \%$. Found: $\mathrm{C}, 75.42 ; \mathrm{H}$, $4.19 ; \mathrm{N}, 5.87 \%$.

(1H-indol-2-yl)(3,4-dimethoxyphenyl)methanone (2h) ${ }^{[2]}$ : White solid, yield: $57 \%$, m.pt. 129-131 ${ }^{\circ} \mathrm{C}$, ( lit. $\left.126-127{ }^{\circ} \mathrm{C}\right),{ }^{1} \mathrm{H}$ NMR (400 MHz, $\left.\mathrm{CDCl}_{3}\right): \delta 10.08$ (br s, $\left.1 \mathrm{H}, \mathrm{NH}\right), 7.70-$ $7.67(\mathrm{t}, J=8 \mathrm{~Hz}, 1 \mathrm{H}), 7.53-7.46(\mathrm{~m}, 3 \mathrm{H}), 7.13-7.10(\mathrm{~d}, J=12 \mathrm{~Hz}, 2 \mathrm{H}), 6.82-6.80(\mathrm{~d}, J=8$ $\mathrm{Hz}, 2 \mathrm{H}), 3.92(\mathrm{~s}, 3 \mathrm{H}), 3.86(\mathrm{~s}, 3 \mathrm{H}) .{ }^{13} \mathrm{C} \mathrm{NMR}\left(100 \mathrm{MHz}, \mathrm{CDCl}_{3}\right): \delta 185.8,152.8,148.8$, $137.5,134.4,130.3,127.6,126.0,124.2,123.9,122.9,120.8,119.9,111.8,109.8,56.0,55.9$. IR $\left(\mathrm{KBr}, v_{\max } \mathrm{cm}^{-1}\right): 3299,1612,1509,1305,1254,1173,1002,839,790,761 . \mathrm{MS}(\mathrm{ESI})$ (m/z) : 282. Anal. calcd. for $\mathrm{C}_{17} \mathrm{H}_{15} \mathrm{NO}_{3}: \mathrm{C}, 72.58 ; \mathrm{H}, 5.37 ; \mathrm{N}, 4.98 \%$. Found: $\mathrm{C}, 72.67 ; \mathrm{H}$, $5.38 ; \mathrm{N}, 4.97 \%$.

(Furan-2-yl)(1H-indol-2-yl)methanone (2i) ${ }^{[1]}$ : Brown solid, yield: 59\%, m.pt. 134-135 ${ }^{\circ} \mathrm{C}$ ( lit. $\left.130-132{ }^{\circ} \mathrm{C}\right),{ }^{1} \mathrm{H}$ NMR (400 MHz, $\mathrm{CDCl}_{3}$ ): $\delta 9.50$ (br s, $\left.1 \mathrm{H}, \mathrm{NH}\right), 7.77$ (dd, $J=16.4,6.7$ $\mathrm{Hz}, 3 \mathrm{H}), 7.54-7.48(\mathrm{~m}, 2 \mathrm{H}), 7.40(\mathrm{t}, J=7.6 \mathrm{~Hz}, 1 \mathrm{H}), 7.20(\mathrm{t}, J=7.2 \mathrm{~Hz}, 1 \mathrm{H}), 6.67(\mathrm{dd}, J=$ 3.4, 1.6 Hz, 1H). ${ }^{13} \mathrm{C}$ NMR (100 MHz, $\left.\mathrm{CDCl}_{3}\right): \delta 172.6,152.7,146.5,137.2,133.4,128.0$, 
126.5, 123.3, 121.0, 118.6, 112.5, 112.1, 111.5. IR (KBr, $\left.v_{\max } \mathrm{cm}^{-1}\right): 3301,1610,1507,1344$, 1275, 1129, 1010, 837, 743. MS(ESI) (m/z) : 212. Anal. calcd. for $\mathrm{C}_{13} \mathrm{H}_{9} \mathrm{NO}_{2}: \mathrm{C}, 73.92 ; \mathrm{H}$, 4.29 ; N, 6.63\%. Found: C, 73.80; H, 4.27; N, 6.62\%.

2-Phenylquinoline (3a) ${ }^{[3]}$ : Oil, yield: 28\%, ${ }^{1} \mathrm{H}$ NMR (400 MHz, $\left.\mathrm{CDCl}_{3}\right): \delta 8.44-8.08(\mathrm{~m}$, 4H), 7.89 (dd, $J=18.8,8.3 \mathrm{~Hz}, 2 \mathrm{H}), 7.77(\mathrm{t}, J=7.6 \mathrm{~Hz}, 1 \mathrm{H}), 7.59-7.51(\mathrm{~m}, 4 \mathrm{H}) .{ }^{13} \mathrm{C} \mathrm{NMR}$ $\left(100 \mathrm{MHz}, \mathrm{CDCl}_{3}\right): \delta 157.4,148.2,139.7,136.8,129.7,129.3,128.8,128.3,127.6,127.4$, 127.2, 126.3, 119.0. IR (KBr, $\left.v_{\max } \mathrm{cm}^{-1}\right): 3053,2958,1710,1596,1551,1446,1282,1074$, 828, 765. MS(ESI) (m/z) : 206. Anal. calcd. for $\mathrm{C}_{15} \mathrm{H}_{11} \mathrm{~N}: \mathrm{C}, 87.77 ; \mathrm{H}, 5.40 ; \mathrm{N}, 6.82 \%$. Found: C, 87.65; H, 5.38; N, 6.81.

2-p-Tolylquinoline (3b) ${ }^{[3]}$ : Light brown solid, yield: $28 \%$, m.pt. $82-84{ }^{\circ} \mathrm{C}$ ( lit. $\left.80-82{ }^{\circ} \mathrm{C}\right),{ }^{1} \mathrm{H}$ NMR (400 MHz, $\left.\mathrm{CDCl}_{3}\right): \delta 8.21(\mathrm{~d}, J=8.5 \mathrm{~Hz}, 2 \mathrm{H}), 8.11(\mathrm{~d}, J=8.1 \mathrm{~Hz}, 2 \mathrm{H}), 7.86(\mathrm{dd}, J=$ 17.5, 8.3 Hz, 2H), 7.75 (t, $J=7.7 \mathrm{~Hz}, 1 \mathrm{H}), 7.54$ (t, $J=7.5 \mathrm{~Hz}, 1 \mathrm{H}), 7.37$ (d, $J=8.0 \mathrm{~Hz}, 2 \mathrm{H})$, 2.47 (s, 3H). ${ }^{13} \mathrm{C} \mathrm{NMR}\left(100 \mathrm{MHz}, \mathrm{CDCl}_{3}\right): \delta 157.3,148.3,139.4,136.8,136.7,129.6,129.6$, 127.4, 127.1, 126.1, 118.9, 21.3. IR $\left(\mathrm{KBr}, \mathrm{v}_{\max } \mathrm{cm}^{-1}\right): 3054,2934,1702,1595,1498,1431$, 1286, 1039, 814, 787. MS(ESI) (m/z) : 220. Anal. calcd. for $\mathrm{C}_{16} \mathrm{H}_{13} \mathrm{~N}: \mathrm{C}, 87.64 ; \mathrm{H}, 5.98 ; \mathrm{N}$, 6.39\%. Found: C, 87.75; H, 5.97; N, 6.41\%.

2-(4-Methoxyphenyl)quinoline (3c) ${ }^{[3]}$ : Light brown solid, yield: $30 \%$, m.pt. $121-123{ }^{\circ} \mathrm{C}$ ( lit.122-124 $\left.{ }^{\circ} \mathrm{C}\right),{ }^{1} \mathrm{H}$ NMR $\left(400 \mathrm{MHz}, \mathrm{CDCl}_{3}\right): \delta 8.18(\mathrm{t}, J=9.3 \mathrm{~Hz}, 4 \mathrm{H}), 7.89-7.79$ (m, 2H), 7.74 (t, $J=7.7 \mathrm{~Hz}, 1 \mathrm{H}), 7.52$ (t, $J=7.4 \mathrm{~Hz}, 1 \mathrm{H}), 7.08$ (d, $J=8.5 \mathrm{~Hz}, 2 \mathrm{H}), 3.91(\mathrm{~s}, 3 \mathrm{H})$. ${ }^{13} \mathrm{C} \mathrm{NMR}\left(100 \mathrm{MHz}, \mathrm{CDCl}_{3}\right): \delta 160.8,156.9,148.2,136.6,132.2,129.6,129.5,128.9,127.4$, 126.9, 125.9, 118.6, 114.2, 55.4. IR $\left(\mathrm{KBr}, \mathrm{v}_{\max } \mathrm{cm}^{-1}\right): 3058,2956,1707,1602,1502,1462$, 1251, 1028, 821, 754. MS(ESI) (m/z) : 236. Anal. calcd. for $\mathrm{C}_{16} \mathrm{H}_{13} \mathrm{NO}: \mathrm{C}, 81.68 ; \mathrm{H}, 5.57$; N, 5.95\%. Found: C, 81.52; H, 5.55; N, 5.94\%. 
2-(3-Methoxyphenyl)quinoline (3d) ${ }^{[3]}$ : Oil, yield: $28 \%,{ }^{1} \mathrm{H}$ NMR $\left(400 \mathrm{MHz}, \mathrm{CDCl}_{3}\right): \delta 8.23$ $(\mathrm{dd}, J=15.2,8.6 \mathrm{~Hz}, 2 \mathrm{H}), 7.88(\mathrm{dd}, J=14.0,8.4 \mathrm{~Hz}, 2 \mathrm{H}), 7.81-7.71(\mathrm{~m}, 3 \mathrm{H}), 7.56(\mathrm{t}, J=$ $7.4 \mathrm{~Hz}, 1 \mathrm{H}), 7.47$ (t, $J=7.9 \mathrm{~Hz}, 1 \mathrm{H}), 7.05(\mathrm{dd}, J=8.2,2.4 \mathrm{~Hz}, 1 \mathrm{H}), 3.97$ (s, 3H). ${ }^{13} \mathrm{C} \mathrm{NMR}$ $\left(100 \mathrm{MHz}, \mathrm{CDCl}_{3}\right): \delta 160.1,157.1,148.2,141.1,136.8,129.8,129.7,129.7,127.3,126.3$, 120.0, 119.1, 115.4, 112.7, 55.4. IR $\left(\mathrm{KBr}, \mathrm{v}_{\max } \mathrm{cm}^{-1}\right): 3059,2932,1712,1599,1557,1467$, 1287, 1041, 830, 781. MS(ESI) (m/z) : 236. Anal. calcd. for $\mathrm{C}_{16} \mathrm{H}_{13} \mathrm{NO}: \mathrm{C}, 81.68 ; \mathrm{H}, 5.57$; N, 5.95\%. Found: C, 81.52; H, 5.59 N, 5.94\%.

2-(4-Chlorophenyl)quinoline (3e) ${ }^{[3]}$ : Brown solid, yield: $29 \%$, m.pt. $110-112^{\circ} \mathrm{C}$ ( lit. $112-$ $\left.113{ }^{\circ} \mathrm{C}\right),{ }^{1} \mathrm{H}$ NMR $\left(400 \mathrm{MHz}, \mathrm{CDCl}_{3}\right): \delta 8.26(\mathrm{~d}, J=8.6 \mathrm{~Hz}, 1 \mathrm{H}), 8.16(\mathrm{dd}, J=13.0,8.6 \mathrm{~Hz}$, 3H), $7.87(\mathrm{dd}, J=8.2,3.9 \mathrm{~Hz}, 2 \mathrm{H}), 7.77(\mathrm{t}, J=7.1 \mathrm{~Hz}, 1 \mathrm{H}), 7.59-7.51(\mathrm{~m}, 3 \mathrm{H}) .{ }^{13} \mathrm{C}$ NMR $\left(100 \mathrm{MHz}, \mathrm{CDCl}_{3}\right): \delta 156.0,148.2,138.0,137.0,135.5,130.9,129.7,129.0,128.8,127.5$, 127.2, 126.5, 118.6. IR (KBr, $\left.v_{\max } \mathrm{cm}^{-1}\right): 3061,2923,1710,1593,1559,1428,1285,1094$, 815, 753. $\mathrm{MS}(\mathrm{ESI})(\mathrm{m} / \mathrm{z}): 240,242$. Anal. calcd. for $\mathrm{C}_{15} \mathrm{H}_{10} \mathrm{NCl}: \mathrm{C}, 75.16 ; \mathrm{H}, 4.21 ; \mathrm{N}$, 5.84\%. Found: C, 75.24; H, 4.20; N, 5.82\%.

2-(4-Bromophenyl)quinoline (3f) ${ }^{[3]}$ : Light brown solid, yield: $27 \%$, m.pt. $120-122{ }^{\circ} \mathrm{C}$ ( lit. 121-123 $\left.{ }^{\circ} \mathrm{C}\right),{ }^{1} \mathrm{H}$ NMR $\left(400 \mathrm{MHz}, \mathrm{CDCl}_{3}\right): \delta 8.25(\mathrm{~d}, J=8.6 \mathrm{~Hz}, 1 \mathrm{H}), 8.18(\mathrm{~d}, J=8.5 \mathrm{~Hz}$, 1H), $8.08(\mathrm{~d}, J=8.2 \mathrm{~Hz}, 2 \mathrm{H}), 7.86(\mathrm{dd}, J=8.4,2.8 \mathrm{~Hz}, 2 \mathrm{H}), 7.77(\mathrm{t}, J=7.7 \mathrm{~Hz}, 1 \mathrm{H}), 7.68(\mathrm{~d}$, $J=8.2 \mathrm{~Hz}, 2 \mathrm{H}), 7.57(\mathrm{t}, J=7.5 \mathrm{~Hz}, 1 \mathrm{H}) .{ }^{13} \mathrm{C} \mathrm{NMR}\left(100 \mathrm{MHz}, \mathrm{CDCl}_{3}\right): \delta 156.0,148.2,138.5$, $137.0,131.9,129.8,129.1,127.5,127.2,126.5,123.9,118.5 . \mathrm{IR}\left(\mathrm{KBr}, v_{\max } \mathrm{cm}^{-1}\right): 3058$, 2923, 1712, 1596, 1551, 1426, 1221, 1072, 818, 767. MS(ESI) (m/z) : 284, 286. Anal. calcd. for $\mathrm{C}_{15} \mathrm{H}_{10} \mathrm{NBr}$ : C, 63.40; H, 3.55; N, 4.93\%. Found: C, 63.53; H, 3.56; N, 4.91\%.

2-(4-Fluorophenyl)quinoline (3g) ${ }^{[3]}$ : Brown solid, yield: $27 \%$, m.pt. $95-96{ }^{\circ} \mathrm{C}$ ( lit. 91$\left.93^{\circ} \mathrm{C}\right),{ }^{1} \mathrm{H}$ NMR $\left(400 \mathrm{MHz}, \mathrm{CDCl}_{3}\right): \delta 8.25(\mathrm{~d}, J=8.6 \mathrm{~Hz}, 1 \mathrm{H}), 8.19(\mathrm{dd}, J=8.8,5.5 \mathrm{~Hz}$, 3H), $7.86(\mathrm{~d}, J=8.5 \mathrm{~Hz}, 2 \mathrm{H}), 7.76(\mathrm{t}, J=7.7 \mathrm{~Hz}, 1 \mathrm{H}), 7.56(\mathrm{t}, J=7.5 \mathrm{~Hz}, 1 \mathrm{H}), 7.24(\mathrm{t}, J=$ 
8.7 Hz, 2H). ${ }^{13} \mathrm{C}$ NMR (100 MHz, $\left.\mathrm{CDCl}_{3}\right): \delta 165.0,162.5,156.2,148.2,136.9,135.8,129.5$, 127.5, 127.0, 126.3, 118.6, 115.9, 115.6. IR (KBr, $\left.v_{\max } \mathrm{cm}^{-1}\right): 3049,2936,1707,1589,1579$, 1434, 1235, 1083, 812, 757. MS(ESI) (m/z) : 224. Anal. calcd. for $\mathrm{C}_{15} \mathrm{H}_{10} \mathrm{NF}: \mathrm{C}, 80.70 ; \mathrm{H}$, $4.51 ; \mathrm{N}, 6.27 \%$. Found: C, 80.83; H, 4.50; N, 6.25\%.

2-(3,4-Dimethoxyphenyl)quinoline (3h): Yellow oil, yield: 29\%, ${ }^{1} \mathrm{H}$ NMR $(400 \mathrm{MHz}$, $\left.\mathrm{CDCl}_{3}\right): \delta 8.23-8.17(\mathrm{~m}, 2 \mathrm{H}), 7.90-7.83(\mathrm{~m}, 2 \mathrm{H}), 7.76-7.68(\mathrm{~m}, 2 \mathrm{H}), 7.55-7.53(\mathrm{~m}, 2 \mathrm{H}), 7.04-$ $7.01(\mathrm{~d}, J=12 \mathrm{~Hz}, 1 \mathrm{H}), 4.08(\mathrm{~s}, 3 \mathrm{H}), 3.97(\mathrm{~s}, 3 \mathrm{H}) .{ }^{13} \mathrm{C} \mathrm{NMR}\left(100 \mathrm{MHz}, \mathrm{CDCl}_{3}\right): \delta 161.0$, $156.8,147.9,136.7,129.6,129.4,127.4,126.9,126.0,123.3,120.2,118.6,111.0,110.3$. IR $\left(\mathrm{KBr}, v_{\max } \mathrm{cm}^{-1}\right): 3052,2942,1702,1599,1512,1457,1245,1035,830,775 . \mathrm{MS}(\mathrm{ESI})(\mathrm{m} / \mathrm{z}):$ 266. Anal. calcd. for $\mathrm{C}_{17} \mathrm{H}_{15} \mathrm{NO}_{2}$ : C, 76.96; H, 5.70; N, 5.28\%. Found: $\mathrm{C}, 77.09 ; \mathrm{H}, 5.69 ; \mathrm{N}$, $5.27 \%$.

2-(Furan-2-yl)quinoline (3i) ${ }^{[4]}$ : Brown solid, yield: $28 \%$, m.pt. $92-94{ }^{\circ} \mathrm{C}$ ( lit. $\left.88-90{ }^{\circ} \mathrm{C}\right),{ }^{1} \mathrm{H}$ NMR (400 MHz, $\left.\mathrm{CDCl}_{3}\right): \delta 8.17(\mathrm{t}, J=9.7 \mathrm{~Hz}, 2 \mathrm{H}), 7.82(\mathrm{dd}, J=15.5,8.4 \mathrm{~Hz}, 2 \mathrm{H}), 7.73(\mathrm{t}$, $J=7.1 \mathrm{~Hz}, 1 \mathrm{H}), 7.66(\mathrm{~s}, 1 \mathrm{H}), 7.52(\mathrm{t}, J=7.1 \mathrm{~Hz}, 1 \mathrm{H}), 7.25(\mathrm{~d}, J=2.7 \mathrm{~Hz}, 1 \mathrm{H}), 6.62(\mathrm{~s}, 1 \mathrm{H})$.

${ }^{13} \mathrm{C}$ NMR $\left(100 \mathrm{MHz}, \mathrm{CDCl}_{3}\right): \delta 153.6,149.0,148.0,144.1,136.7,129.9,129.3,127.6,127.1$, 126.2, 117.5, 112.2, 110.2. IR (KBr, $\left.v_{\max } \mathrm{cm}^{-1}\right): 3059,2945,1710,1613,1526,1489,1242$, 1057, 820, 719. $\mathrm{MS}(\mathrm{ESI})(\mathrm{m} / \mathrm{z})$ : 196. Anal. calcd. for $\mathrm{C}_{13} \mathrm{H}_{9} \mathrm{NO}: \mathrm{C}, 79.98 ; \mathrm{H}, 4.65 ; \mathrm{N}$, 7.17\%. Found: C, 80.09; H, 4.63; N, 7.19\%.

\section{References}

1) Zhao, Y.; Li, D.; Zhao, L.; Zhang, J. Synthesis 2011, 873-880.

2) Takeda, Y.; Kikuchi, A.; Terashima, M. Heterocycles 1993, 35, 573-576.

3) Han, R.; Chen, S.; Lee, S. J.; Qi, F.; Wu, X.; Kim, B. H. Heterocycles 2006, 68, $1675-$ 1684. 
4) Cho, C. S.; Kim, B. T.; Choi, H. -J.; Kim, T. -J.; Sim, S. C. Tetrahedron 2003, 59, 7997-8002. 
(1H-indol-2-yl)(phenyl)methanone (2a)

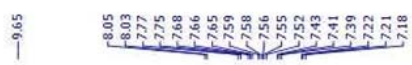
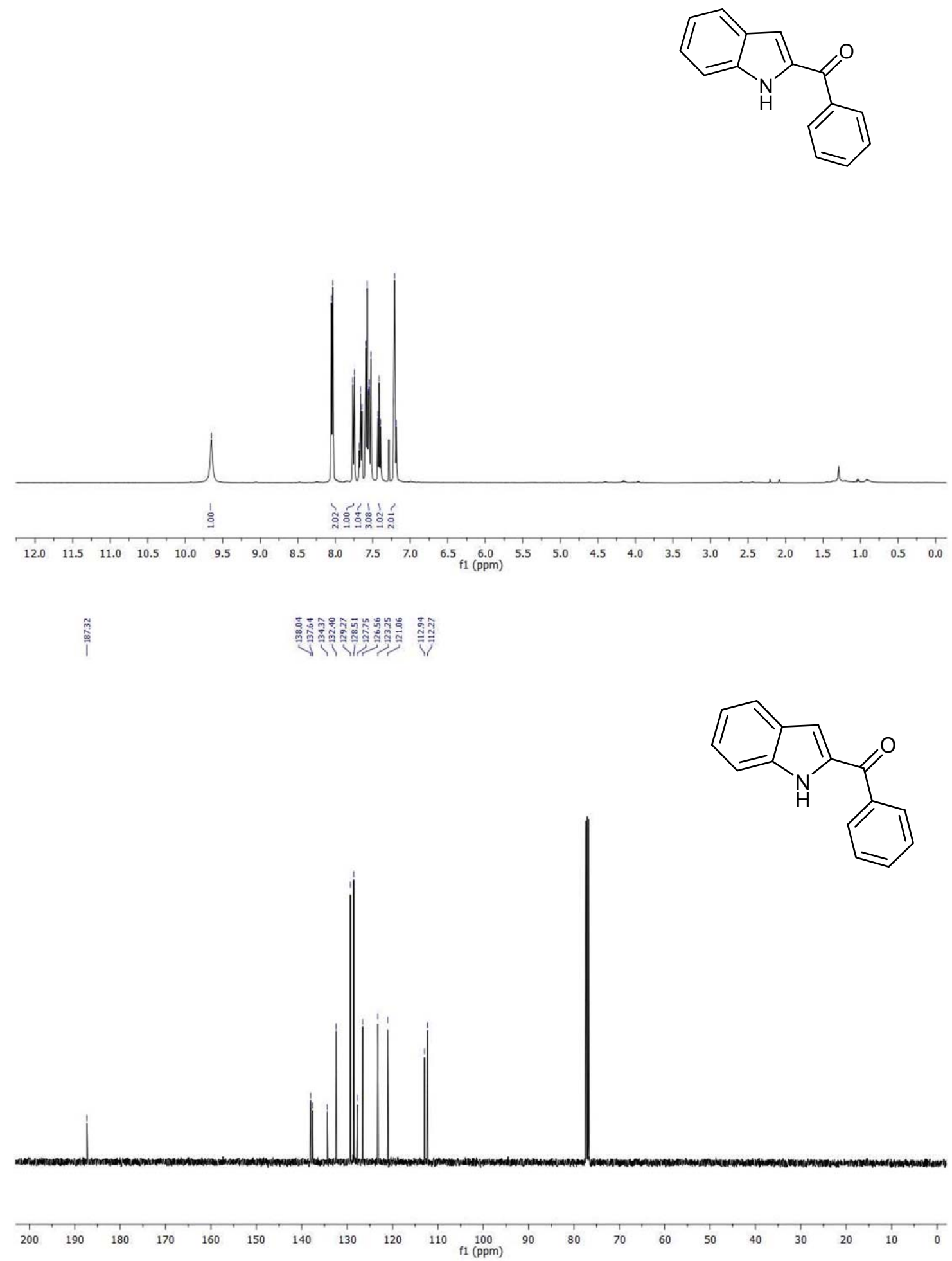
(1H-indol-2-yl)(p-tolyl)methanone (2b)

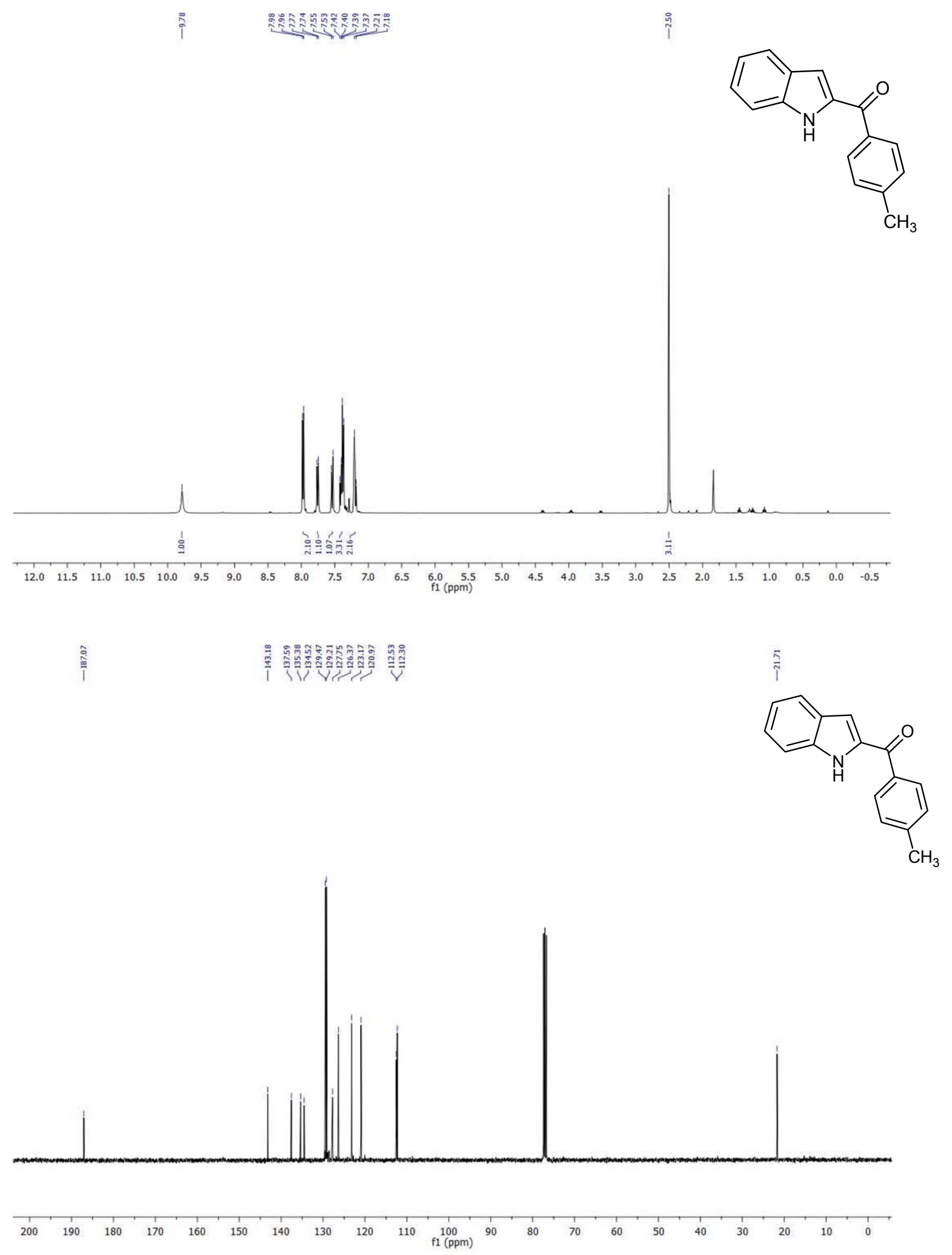


(1H-indol-2-yl)(4-methoxyphenyl)methanone (2c)
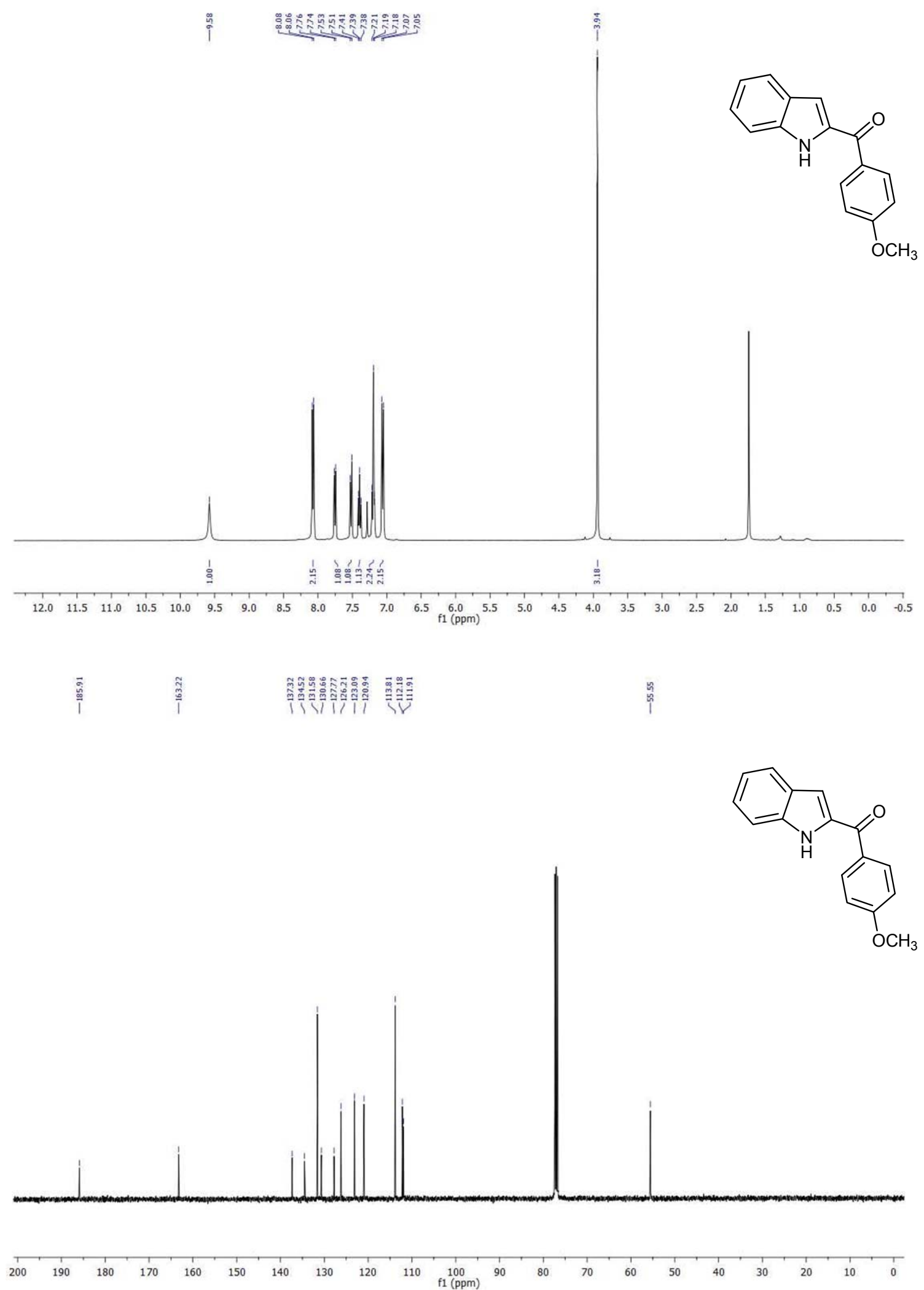
(1H-indol-2-yl)(3-methoxyphenyl)methanone (2d)
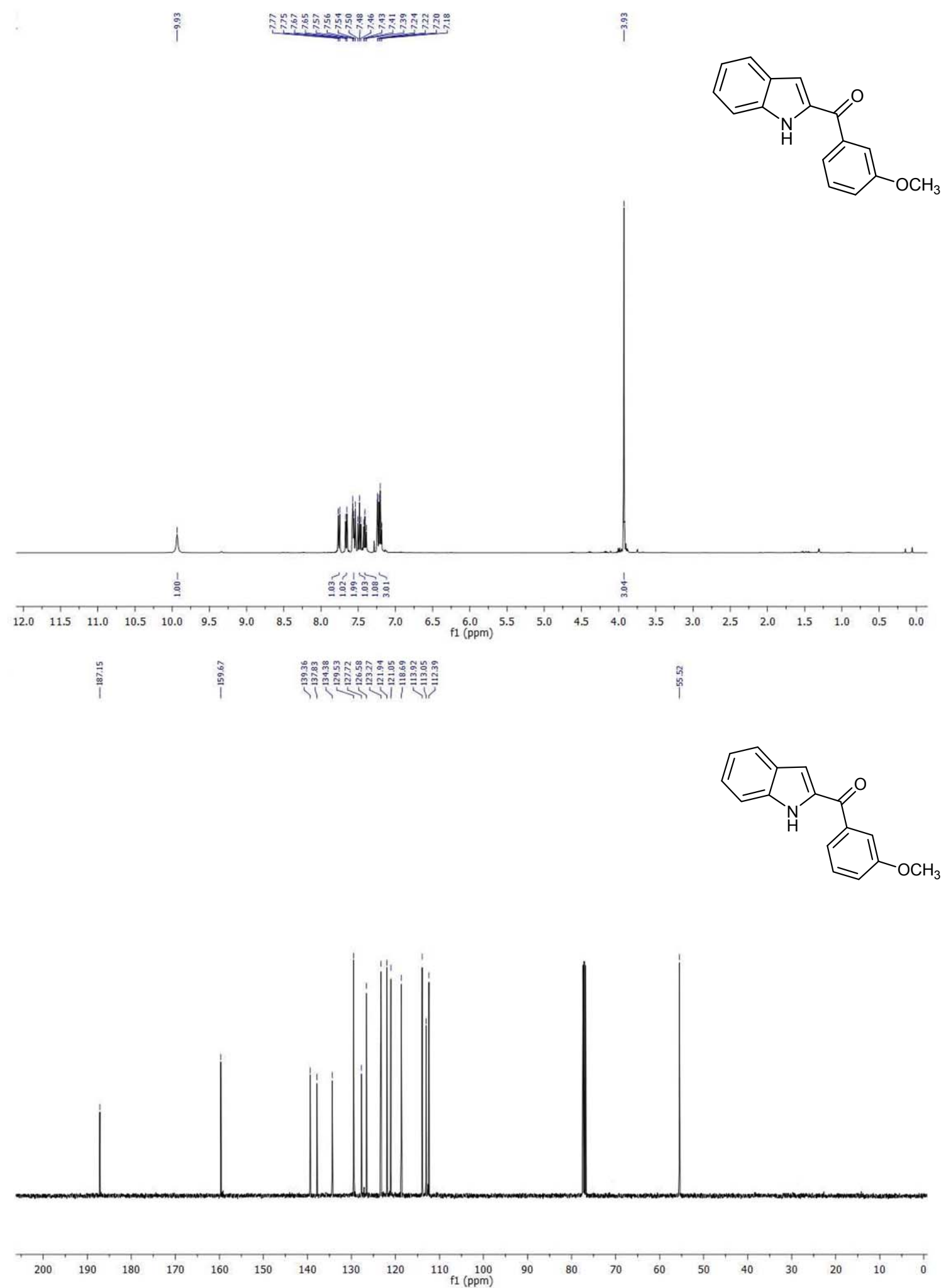
(4-Chlorophenyl)(1H-indol-2-yl)methanone (2e)

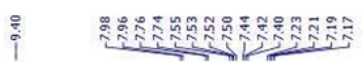
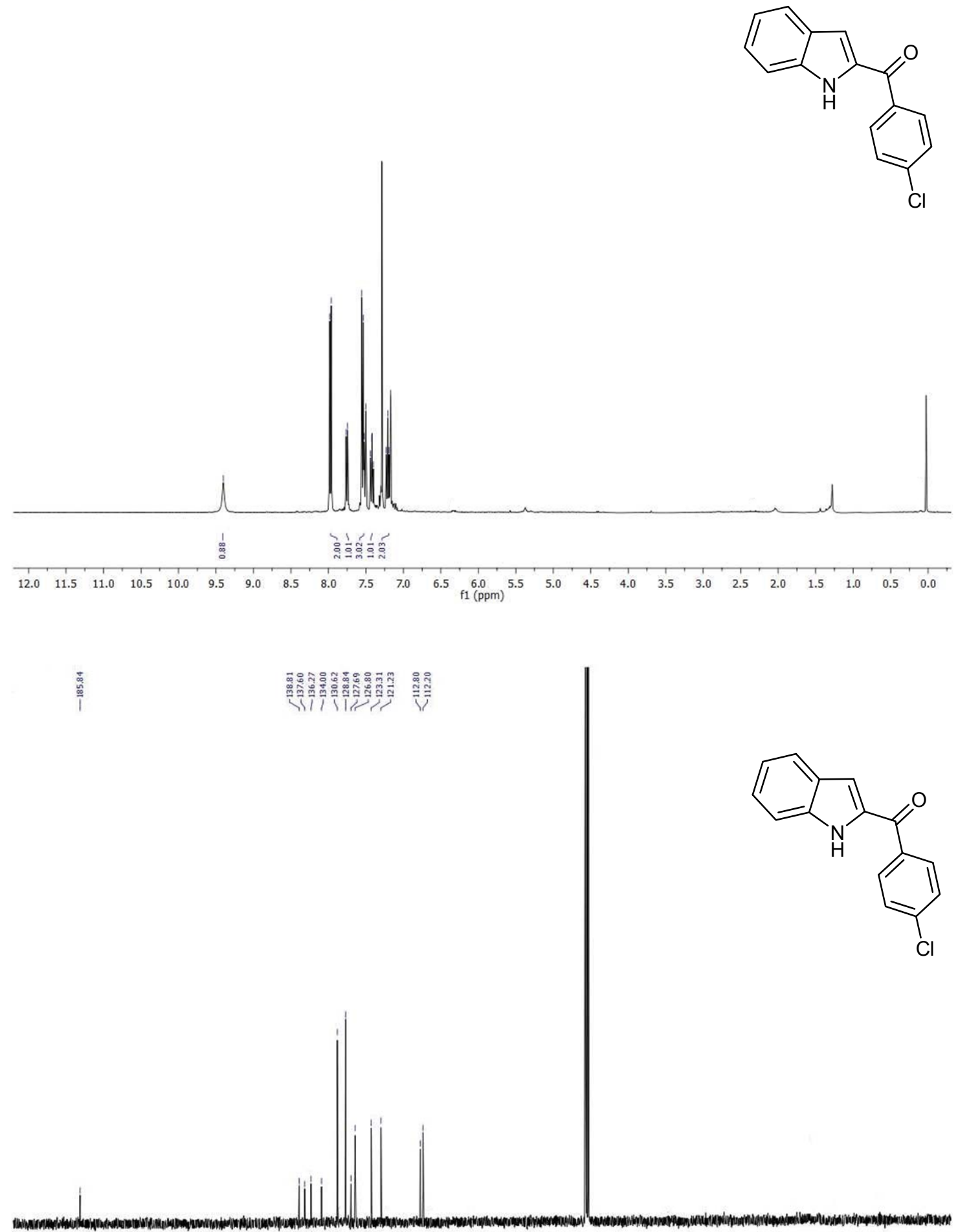

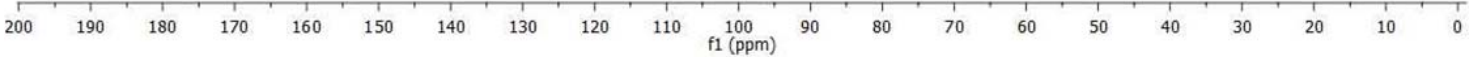


(4-Bromonhenvl)(1H-indol-2-vl)methanone (2f)

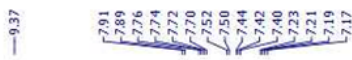
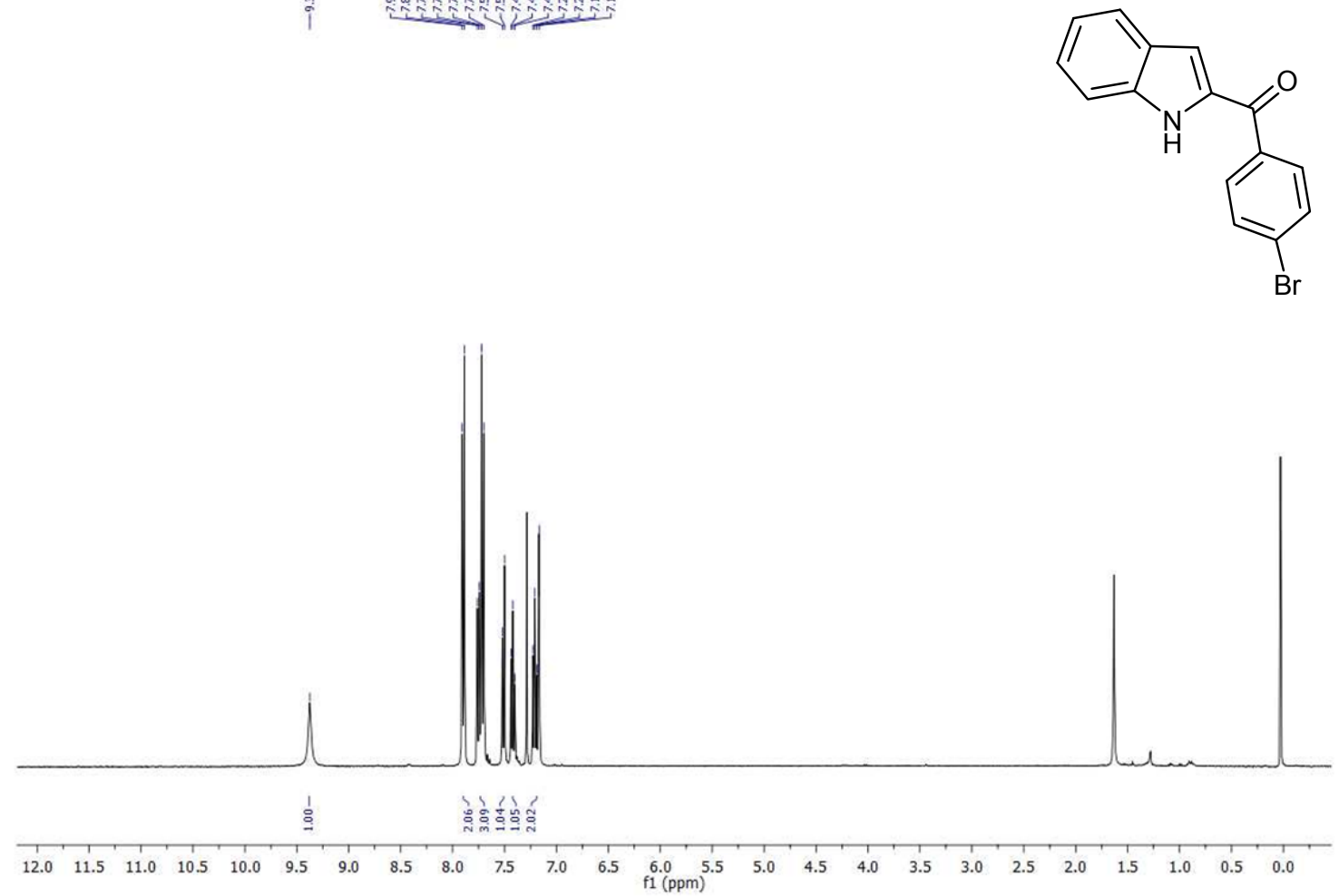

|
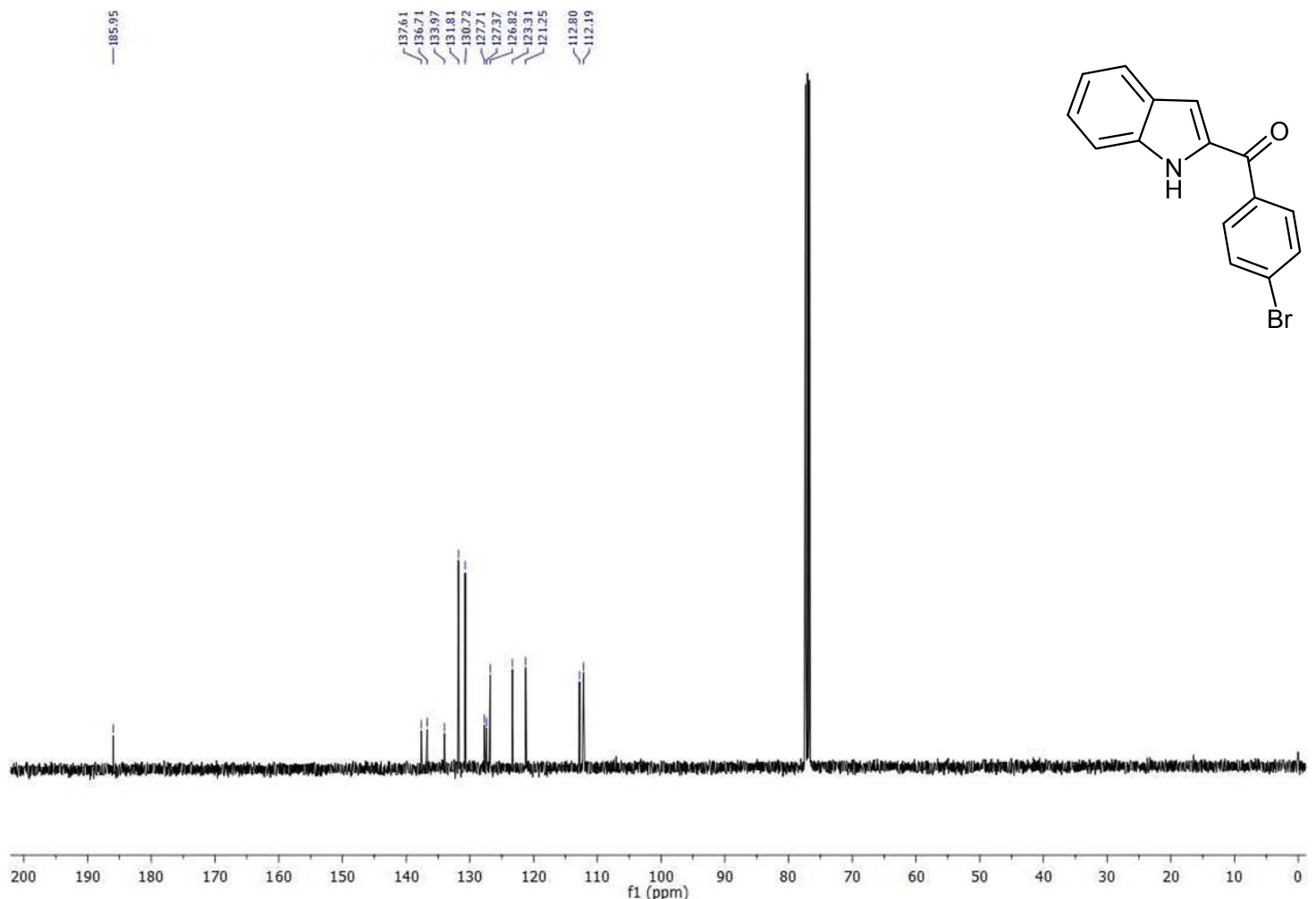
(4-Fluorophenyl)(1H-indol-2-yl)methanone (2g)
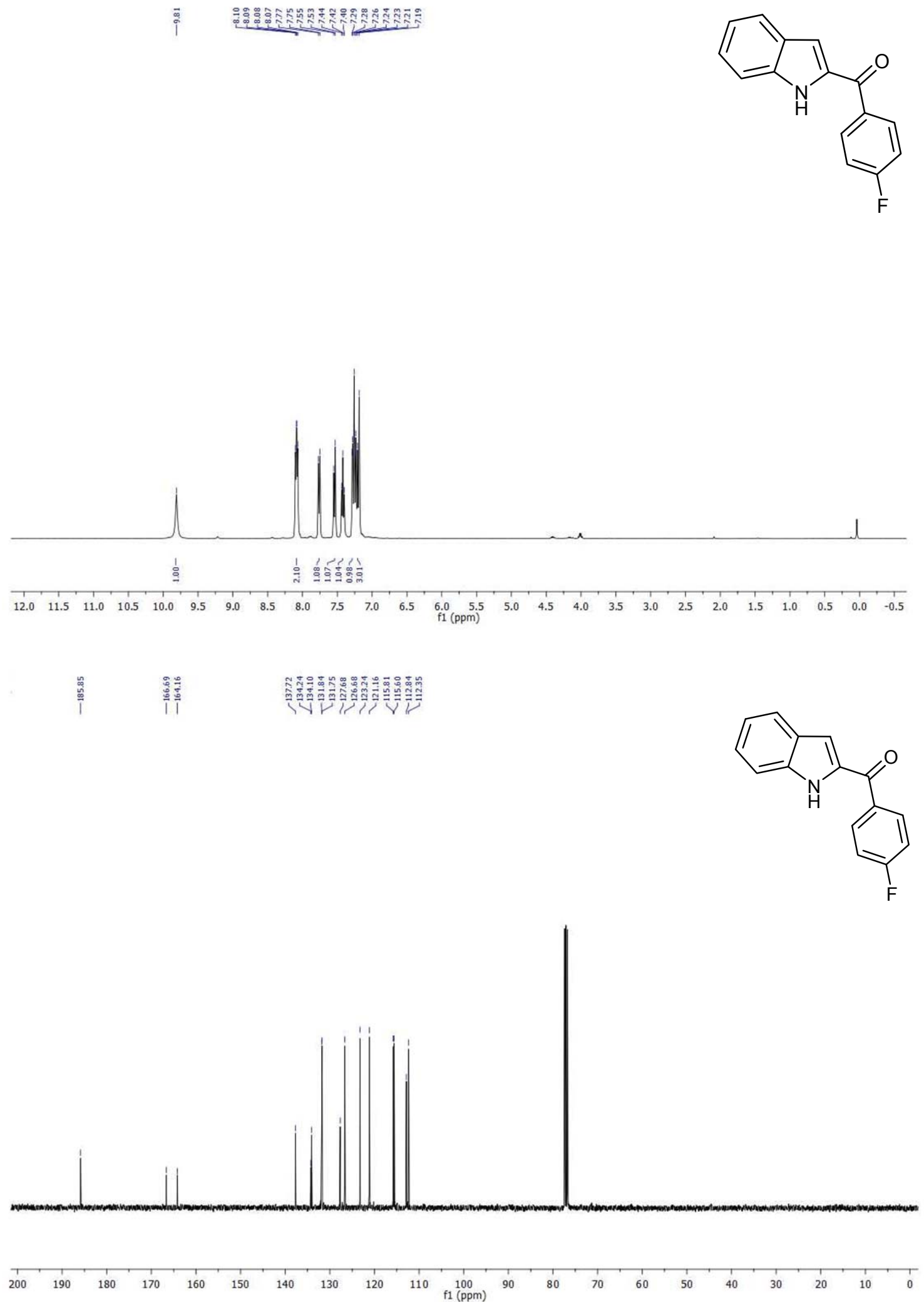
(1H-indol-2-yl)(3,4-dimethoxyphenyl)methanone (2h)
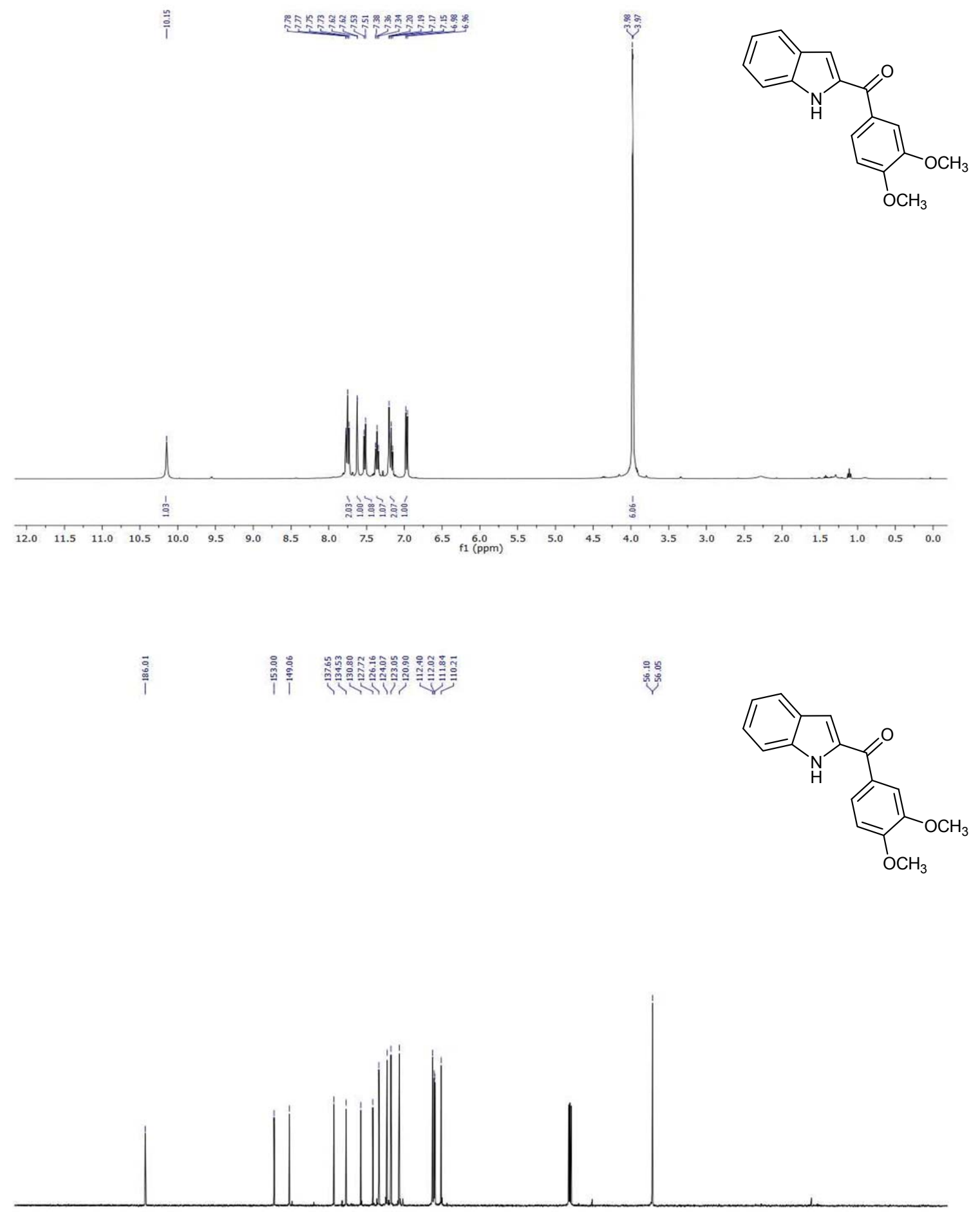

$\begin{array}{llllllllllllllllllllllll}210 & 200 & 190 & 180 & 170 & 160 & 150 & 140 & 130 & 120 & 110 & 100 & 90 & 80 & 70 & 60 & 50 & 40 & 30 & 20 & 10 & 0 & -10 & 10\end{array}$ 
(Furan-2-yl)(1H-indol-2-yl)methanone (2i):
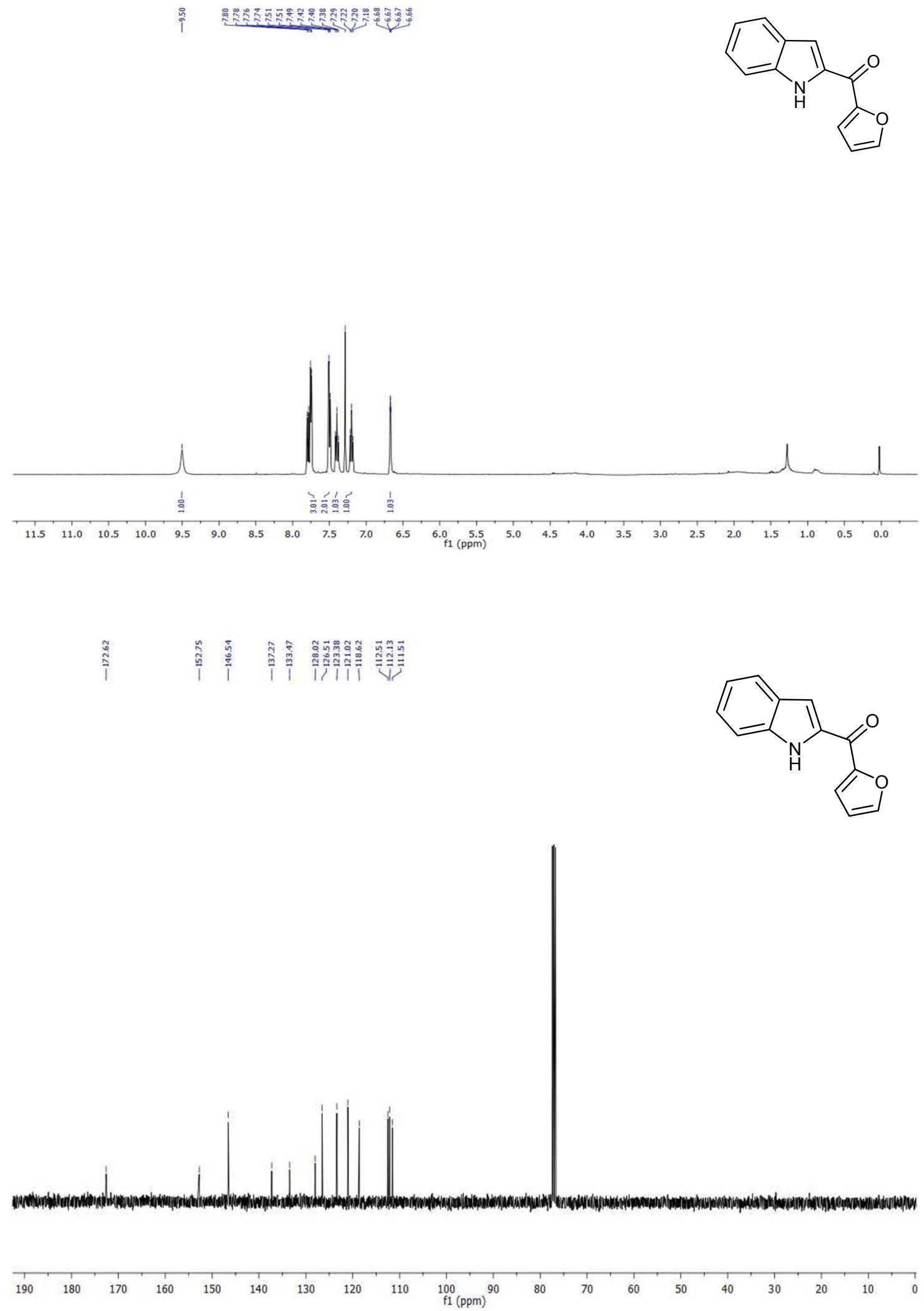
2-Phenylquinoline (3a)

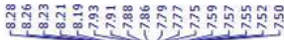
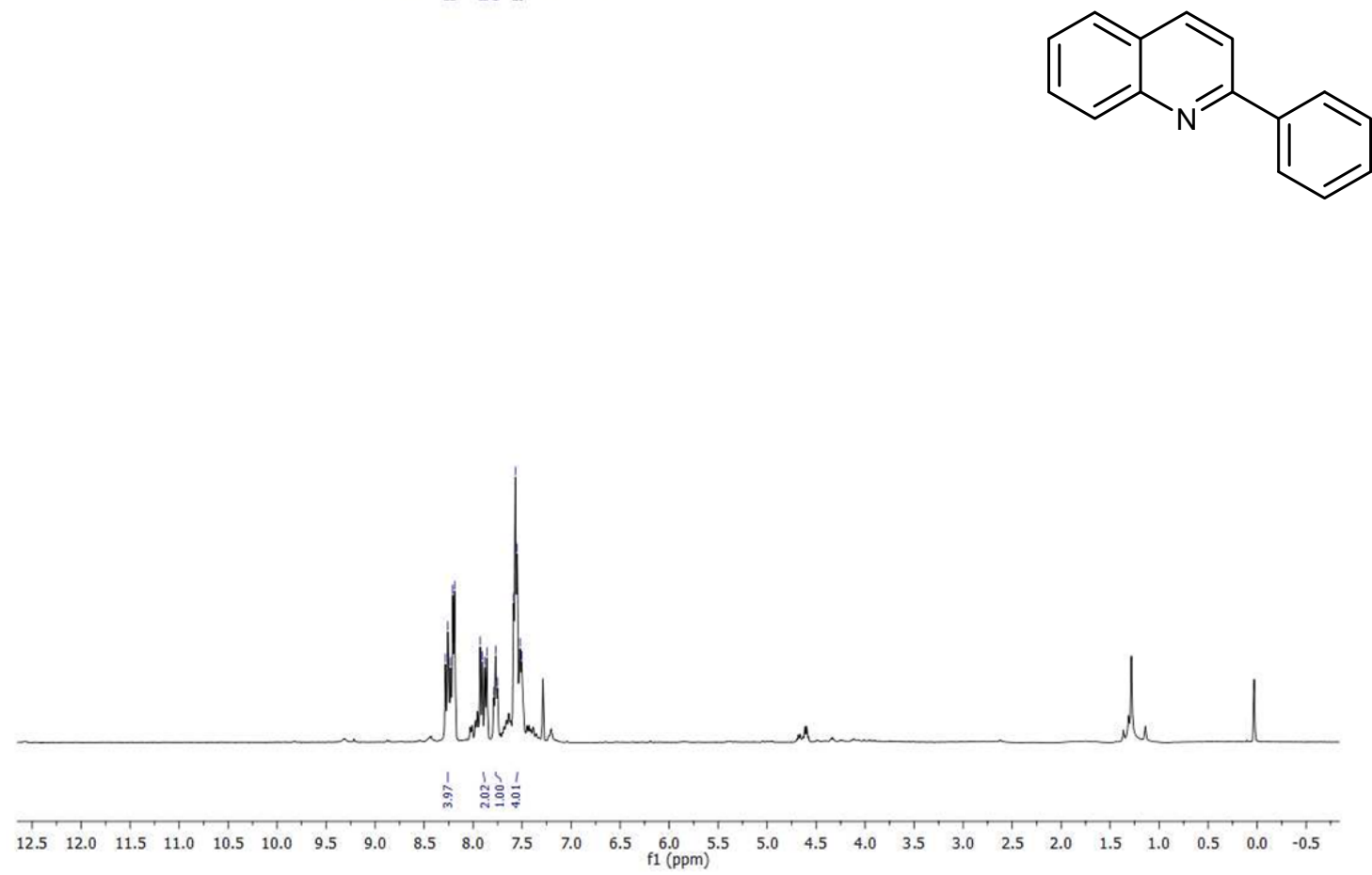

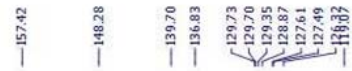

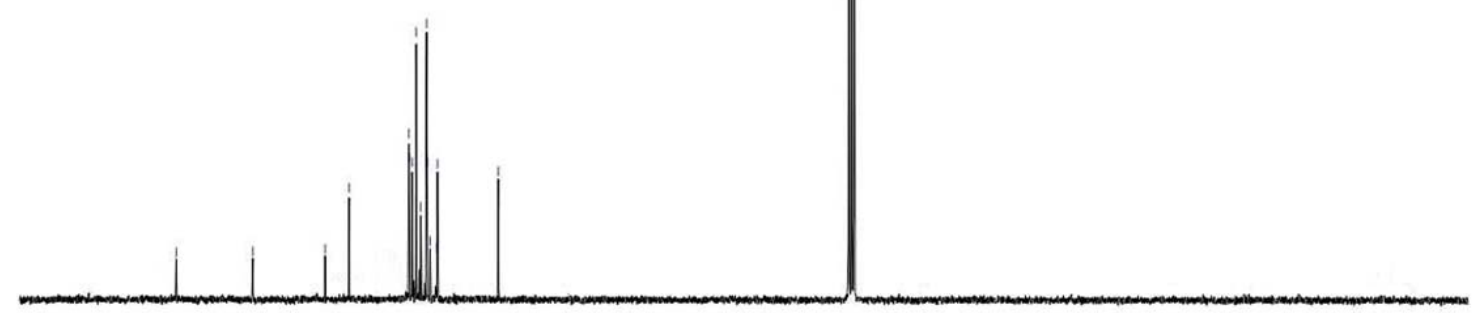


2-p-Tolylquinoline (3b)

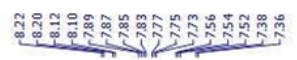

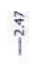
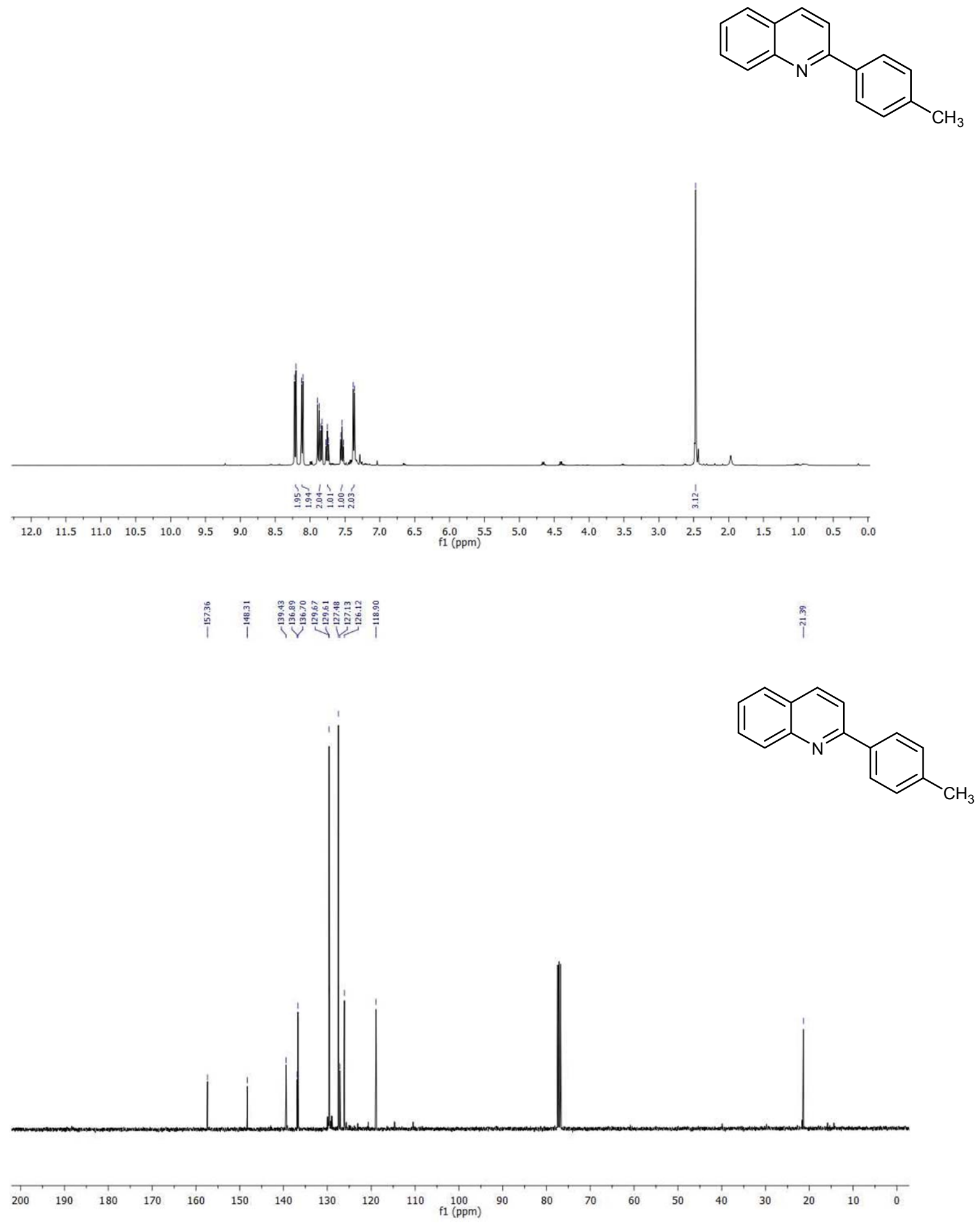

19 
2-(4-Methoxyphenyl)quinoline (3c)

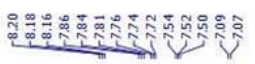

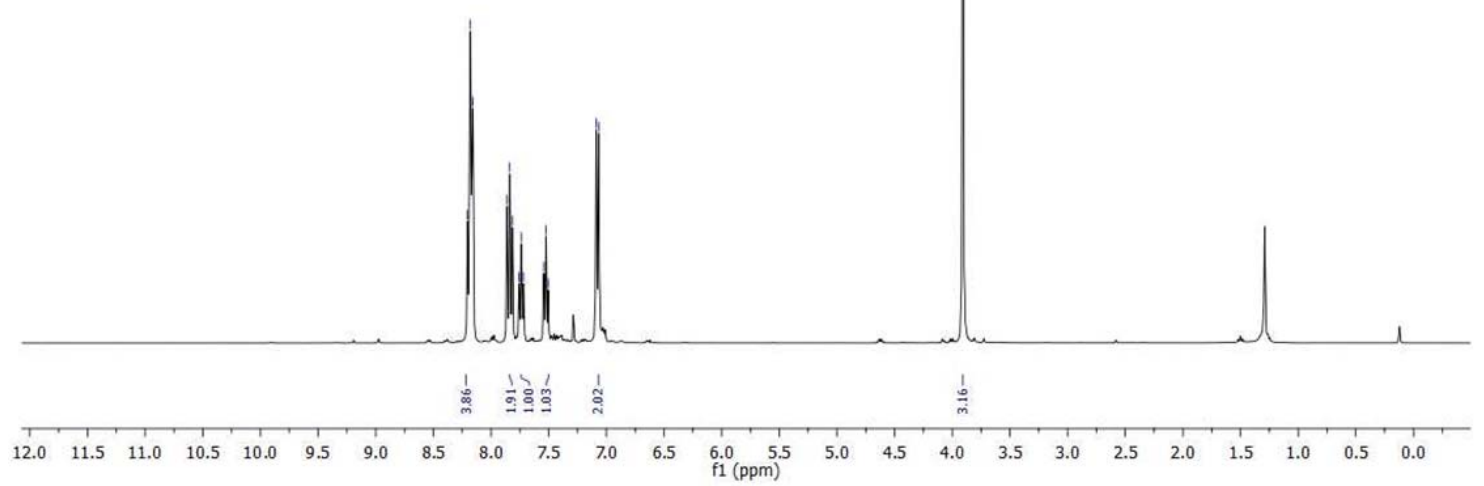

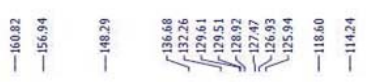

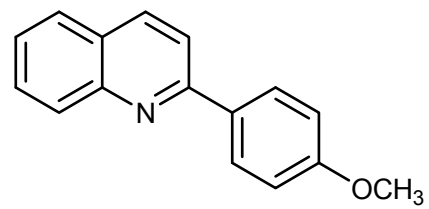

草
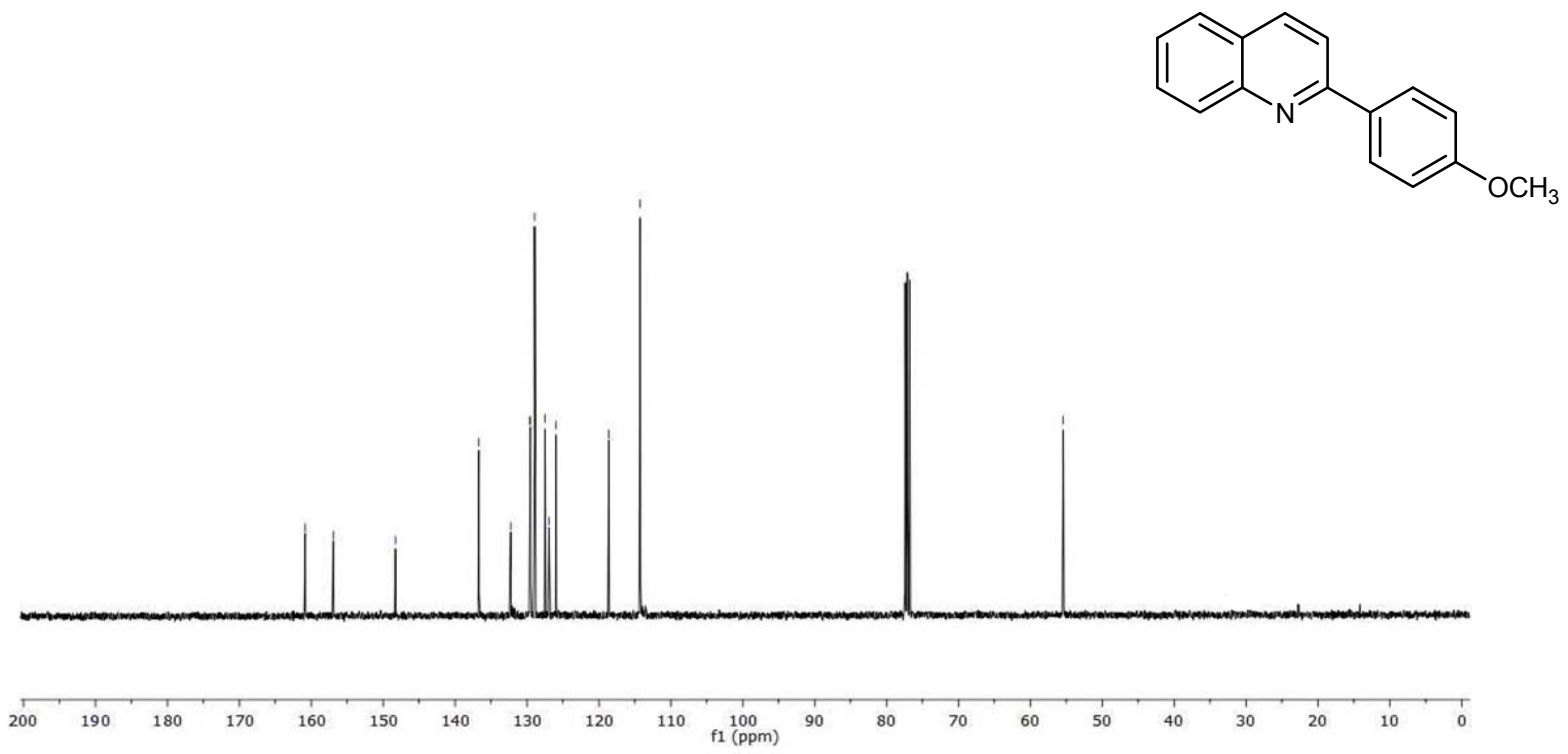

20 
2-(3-Methoxyphenyl)quinoline (3d)

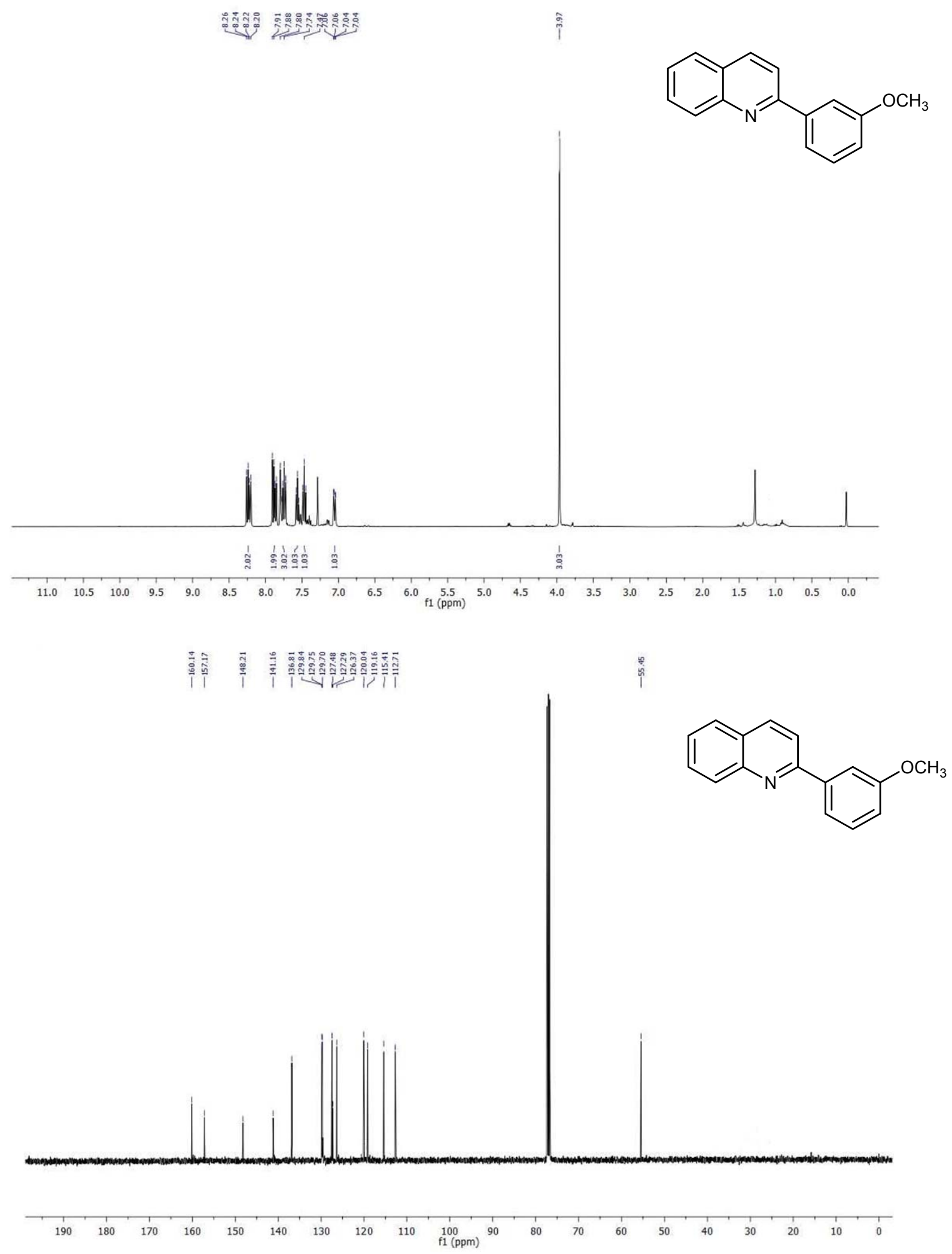


2-(4-Chlorophenyl)quinoline (3e)

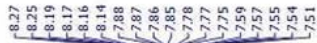
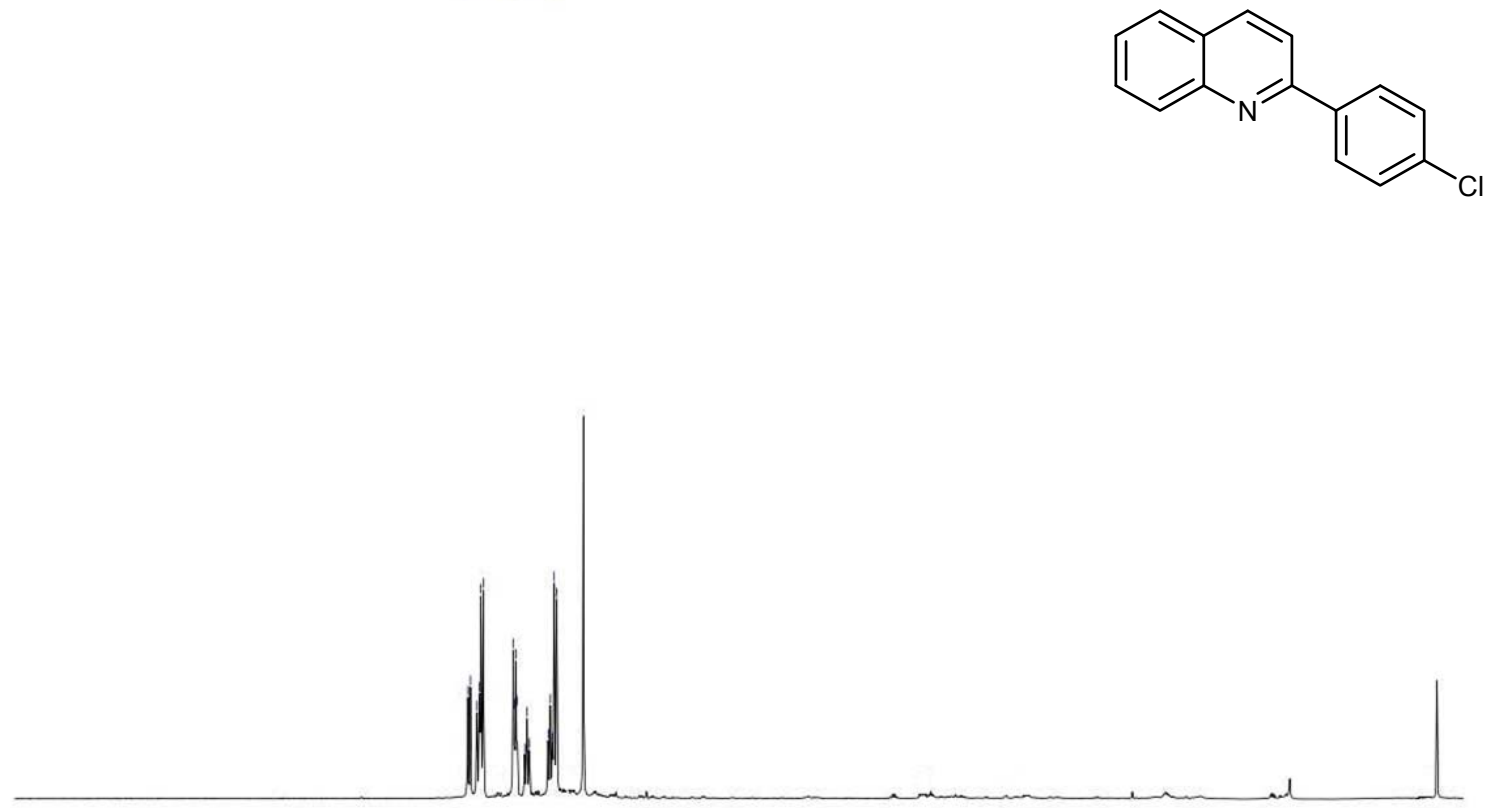

解
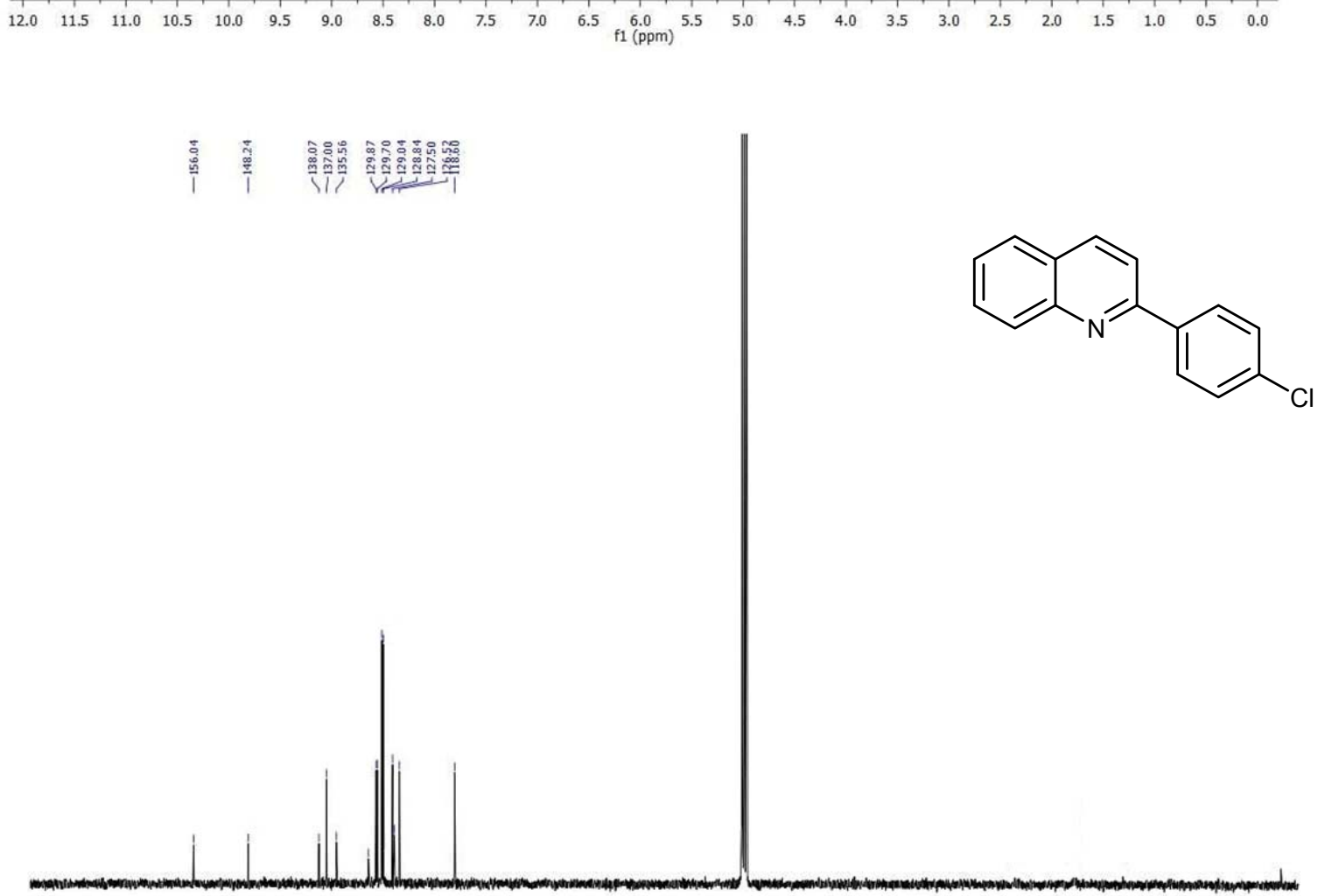
2-(4-Bromophenyl)quinoline (3f)

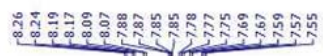
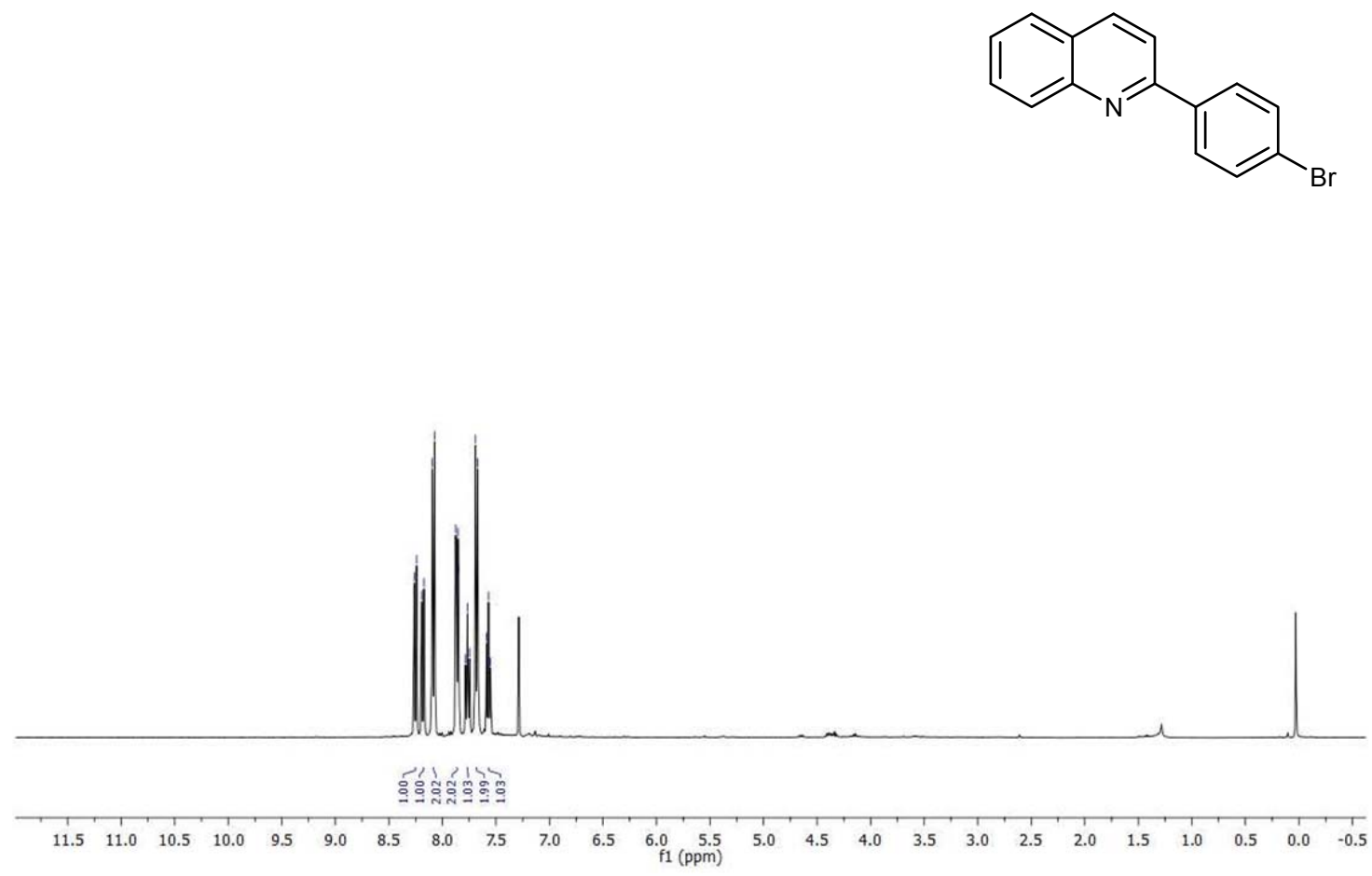

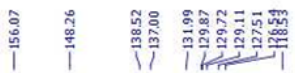

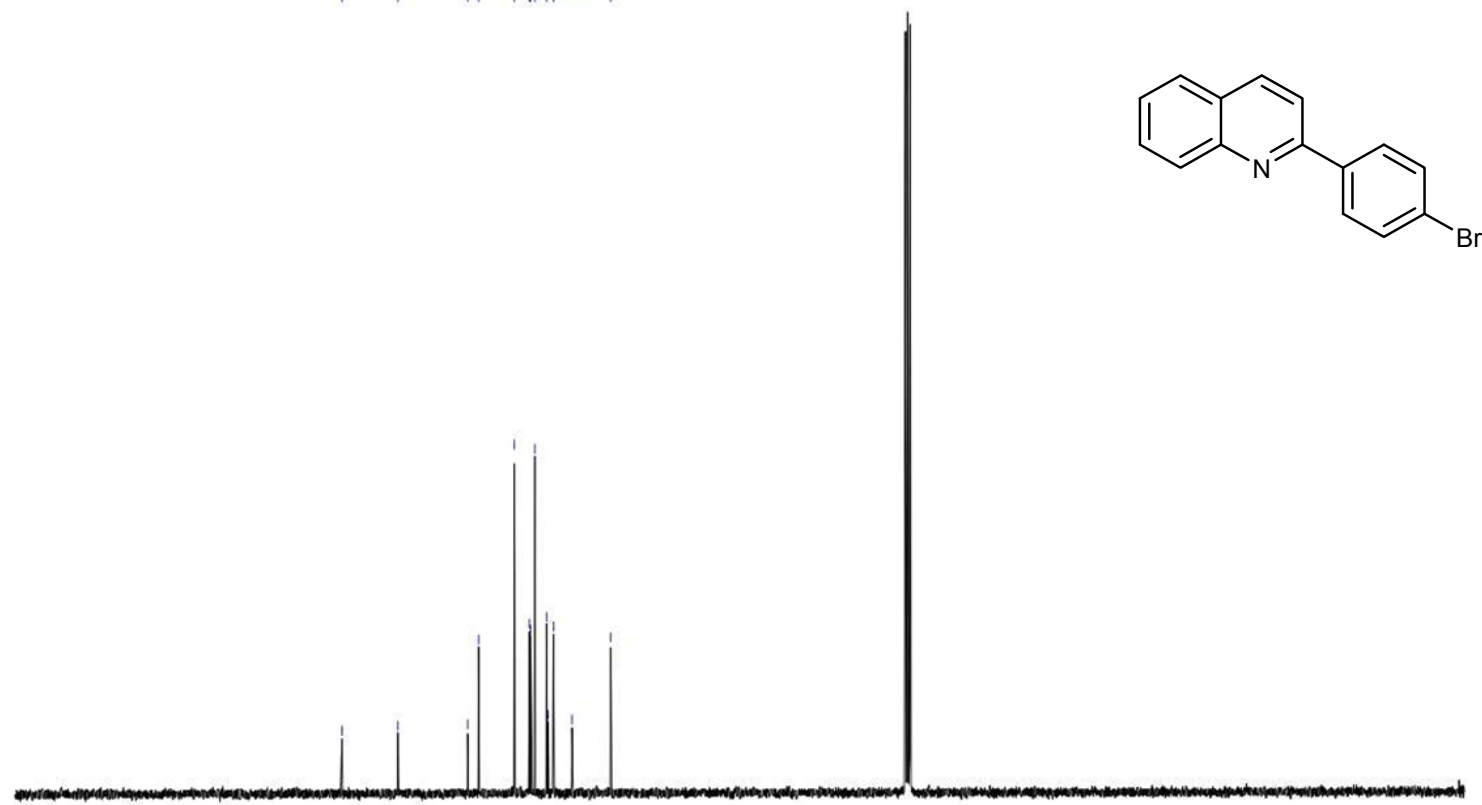

$\begin{array}{lllllllllllllllllllll}200 & 190 & 180 & 170 & 160 & 150 & 140 & 130 & 120 & 110 & \begin{array}{c}100 \\ 100\end{array} & 90 & 80 & 70 & 60 & 50 & 40 & 30 & 20 & 10 & 0\end{array}$ 
2-(4-Fluorophenyl)quinoline (3g)

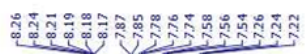
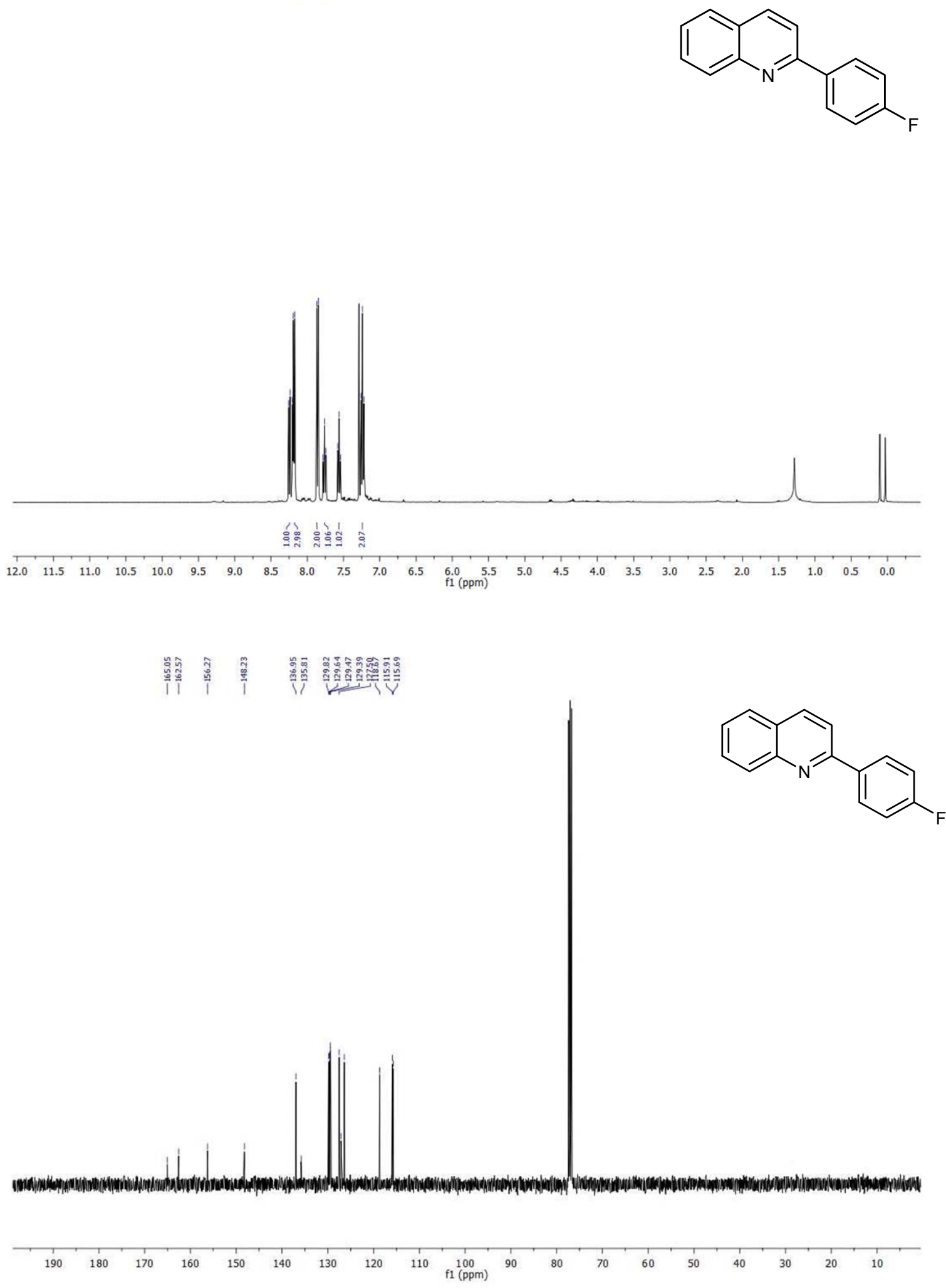

24 
2-(3,4-Dimethoxyphenyl)quinoline (3h)

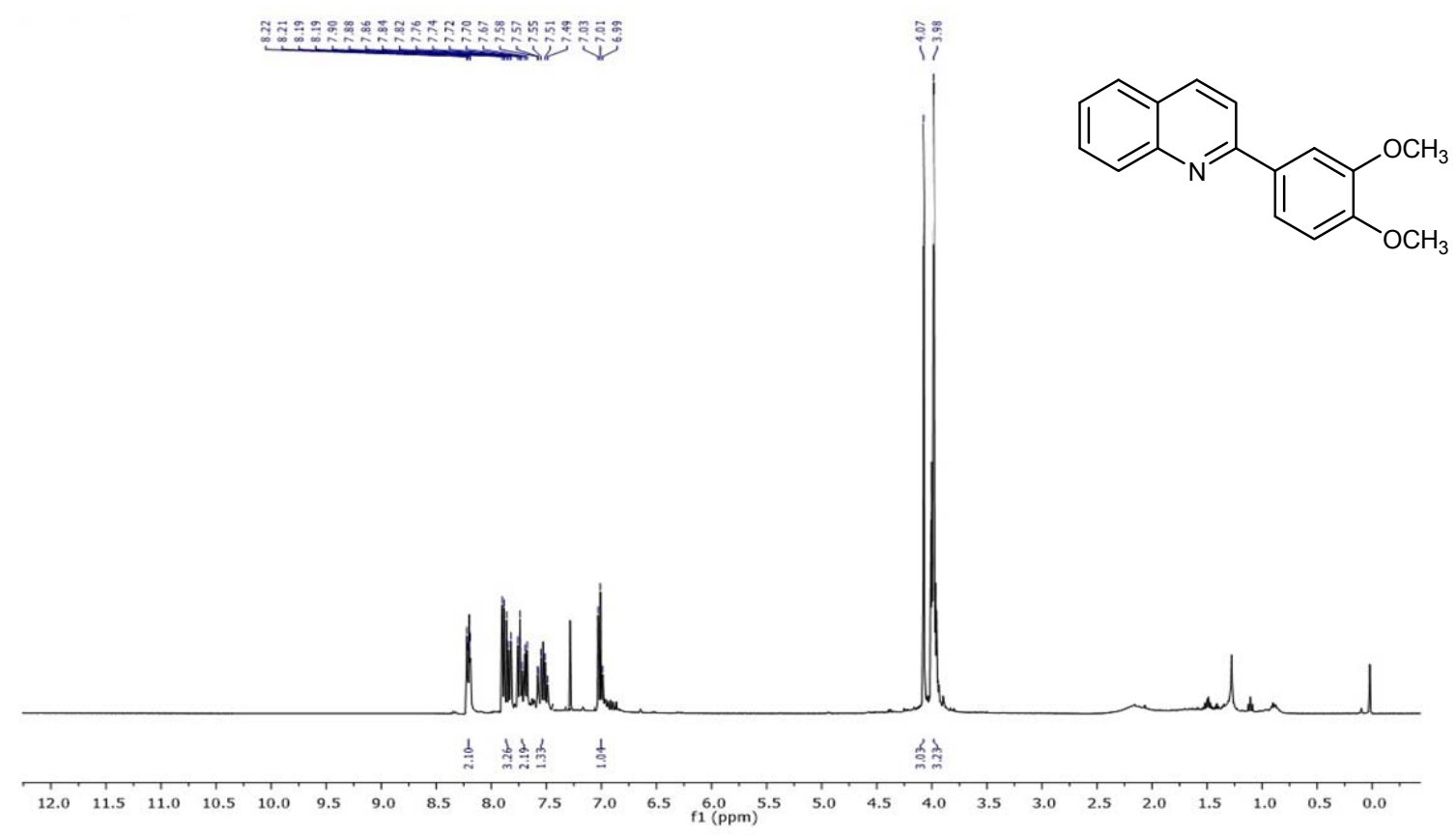

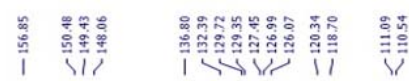

ชั้<smiles>COc1ccc(-c2ccc3ccccc3n2)cc1OC</smiles>

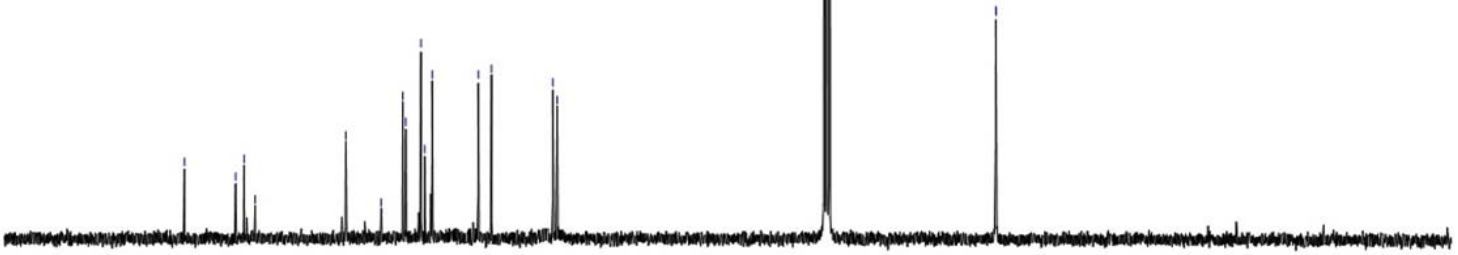

17

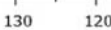

110

100

$\mathrm{g}_{1} \stackrel{90}{(\mathrm{ppm})}$
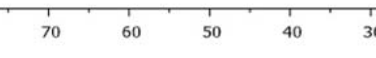
2-(Furan-2-yl)quinoline (3i)

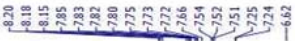
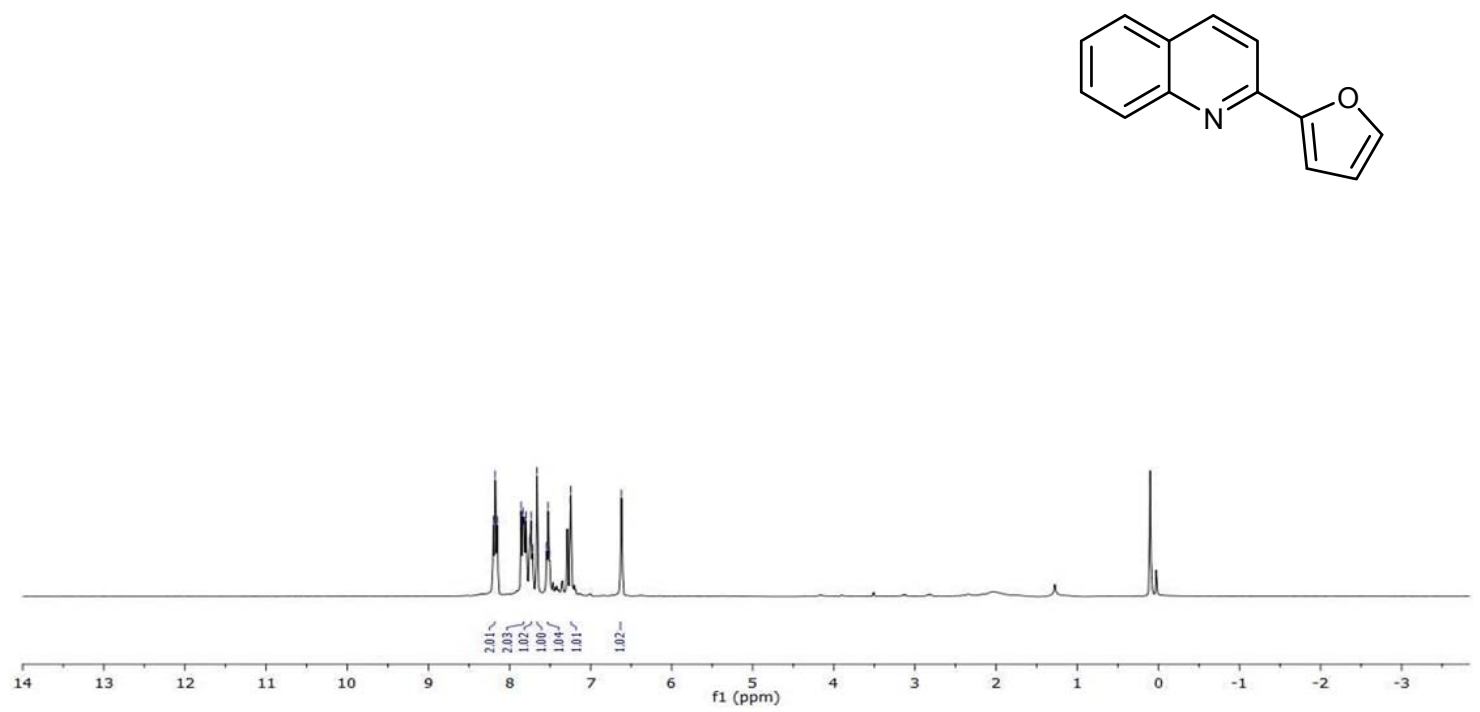

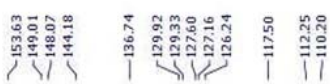

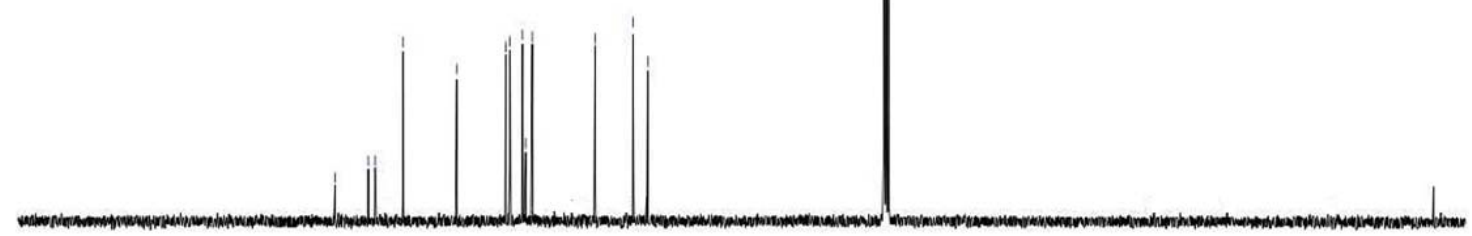

